\title{
GALEX OBSERVATIONS OF LOW SURFACE BRIGHTNESS GALAXIES: UV COLOR AND STAR FORMATION EFFICIENCY ${ }^{1}$
}

\author{
S. Boissier, ${ }^{2,3}$ A. Gil de Paz, ${ }^{4}$ A. Boselli, ${ }^{2,3}$ V. Buat, ${ }^{2}$ B. Madore, ${ }^{5}$ L. Chemin, ${ }^{6}$ \\ C. Balkowski, ${ }^{6}$ P. Amram, ${ }^{2}$ C. Carignan, ${ }^{7}$ and W. van Driel ${ }^{6}$ \\ Received 2008 January 21; accepted 2008 March 20
}

\begin{abstract}
We present GALEX UV observations of a sample of low surface brightness (LSB) galaxies for which H i data are available, allowing us to estimate their star formation efficiency. We find that the UV light extends to larger radii than the optical light (some galaxies, but not all, look similar to the recently discovered XUV-disk galaxies). Using a standard calibration to convert the UV light into star formation rates, we obtain lower star formation efficiencies in LSB galaxies than in high surface brightness galaxies by about one order of magnitude. We show, however, that standard calibrations may not apply to these galaxies, as the FUV-NUV color obtained from the two GALEX bands (FUV and NUV; $\lambda_{\text {eff }}=1516$ and $2267 \AA$, respectively) is redder than expected for star-forming galaxies. This color can be interpreted as a result of internal extinction, modified initial mass function, or star formation histories characterized by bursts followed by quiescent phases. Our analysis favors this latter hypothesis.
\end{abstract}

Subject headings: galaxies: dwarf — galaxies: irregular — galaxies: spiral — ultraviolet: galaxies

Online material: color figures, extended figure set

\section{INTRODUCTION}

In the very last years, outskirts of galaxies and low-density regions have been the subject of a renewed interest, especially after the discovery of extended ultraviolet (XUV) disks in nearby galaxies with GALEX (Gil de Paz et al. 2005; Thilker et al. 2005). The works by Zaritsky \& Christlein (2007) and Thilker et al. (2007) suggests that about $30 \%$ of disk galaxies do present some level of XUV emission. Boissier et al. (2007) showed that the UV reveals stellar formation in the outskirts of normal galaxy disks, including regions beyond the usual "threshold" for star formation derived from $\mathrm{H} \alpha$ azimuthally averaged profiles (Martin \& Kennicutt 2001). The ultraviolet GALEX observations also allowed estimation of the amount of star formation in low-density regions in the case of interactions. For instance, Boselli et al. (2005) discovered in NGC 4438 a tidal tail detected only in UV, and estimated the age and the amplitude of the burst induced by the interaction. Similarly, Boquien et al. (2007) studied star formation in the intergalactic medium around NGC 5291, expelled from parent galaxies after a collision.

As noted by Thilker et al. (2007), a link between the XUV phenomenon, star formation in low-density regions, and low surface brightness (LSB) galaxies is very likely. According to a handwaving definition, a disk galaxy should be considered as a LSB galaxy if its disk central face-on surface brightness in the $B$ band

\footnotetext{
${ }^{1}$ Based on observations made with the NASA Galaxy Evolution Explorer. GALEX is operated for NASA by the California Institute of Technology under NASA contract NAS5-98034.

${ }_{2}^{2}$ Laboratoire d'Astrophysique de Marseille, Pôle de l'Étoile Site de ChâteauGombert, 38, rue Frédéric Joliot-Curie, 13388 Marseille cedex 13, France.

3 CNRS, UMR 6110.

4 Department de Astrofisica y CC. de la Atmosfera, Universidad Complutense de Madrid, Avda. de la Complutense, s/n Madrid, E-28040, Spain.

${ }_{5}^{5}$ Observatories of the Carnegie Institution of Washington, 813 Santa Barbara Street, Pasadena, CA 91101.

6 Observatoire de Paris, GEPI, CNRS and Université Paris 7, 5 Place Jules Janssen, 92195 Meudon Cedex, France.

7 Université de Montréal, CP 6128, Succ center-ville, Montréal, P. QC H3C3J7, Canada.
}

is well below the typical Freeman value of $21.65 \mathrm{mag} \operatorname{arcsec}^{-2}$ (see, e.g., Bothun et al. 1997). LSB galaxies include quite different populations, ranging from dwarf galaxies (faint and relatively compact objects) to disk galaxies, and even "giant" disk galaxies with scale lengths larger than $5 \mathrm{kpc}$ and masses comparable to the more massive spirals (O'Neil et al. 1998). Various samples of "LSB galaxies" do not always trace the same population, and one should take this fact into account. In this paper, we will distinguish "massive" and "low-mass" LSB galaxies according to the $\mathrm{H}$ I total mass, since we have this quantity for every object in our sample.

LSB galaxies have in general faint surface brightnesses, blue colors (see, e.g., de Blok et al. 1995), large amounts of neutral gas (O'Neil et al. 1998), and low metallicity (McGaugh 1994). Similarly, XUV regions have low metallicities (Gil de Paz et al. 2007a) and are found in galaxies that are systematically more gas-rich than the general field galaxy population (Thilker et al. 2007). Star formation in XUV disks and LSB galaxies is thus likely to share some characteristics.

Several models to explain the existence and properties of LSB galaxies have been proposed (e.g., Jimenez et al. 1998; van den Hoek et al. 2000; Gerritsen \& de Blok 1999; Boissier et al. 2003). All of them rely on the inclusion of a recipe for star formation, often characterized by a lower efficiency than in high surface brightness (HSB) galaxies, related to either structural parameters (larger size and lower densities) or metallicity. Characterizing the star formation in LSB galaxies is thus an important step that will set constraints on their modeling. Star formation rates in LSB galaxies have been derived from their optical properties (e.g., McGaugh \& Bothun 1994; van den Hoek et al. 2000), and a few attempts to measure them can be found in Burkholder et al. (2001), van Zee et al. (1997), and O'Neil et al. (2007). None of these studies is based on UV data, while GALEX has shown the promise of the ultraviolet domain to reveal star formation in low-density regions.

On the basis of these considerations, we observed with GALEX a sample of LSB galaxies for which $\mathrm{H}$ I data are available. This paper presents the results of a first analysis of these 
TABLE 1

Basic Properties of the Sample

\begin{tabular}{|c|c|c|c|c|c|c|c|c|}
\hline Name & $\begin{array}{l}\text { R.A. } \\
\text { (deg) }\end{array}$ & $\begin{array}{l}\text { Decl. } \\
\text { (deg) }\end{array}$ & $\begin{array}{c}2 \mathrm{a} \\
(\operatorname{arcmin})\end{array}$ & $\begin{array}{c}2 \mathrm{~b} \\
(\operatorname{arcmin})\end{array}$ & $\begin{array}{l}\text { P.A. } \\
\text { (deg) }\end{array}$ & $\begin{array}{l}\text { Distance } \\
(\mathrm{Mpc})\end{array}$ & $\begin{array}{c}\log M(\mathrm{H} \mathrm{I}) \\
\left(M_{\odot}\right)\end{array}$ & $\begin{array}{c}M_{B} \\
(\mathrm{mag})\end{array}$ \\
\hline UGC $00568 \ldots \ldots \ldots \ldots \ldots \ldots$ & 13.787 & -1.046 & 1.3 & 0.9 & 0 & 190.0 & 10.25 & -21.53 \\
\hline UGC $01230 \ldots \ldots \ldots \ldots \ldots \ldots$ & 26.385 & 25.521 & 2.1 & 1.8 & -68 & 49.2 & 9.76 & $\ldots$ \\
\hline UGC $02936 \ldots \ldots \ldots \ldots \ldots \ldots$ & 60.701 & 1.966 & 2.5 & 0.7 & 30 & 51.2 & 9.85 & -19.58 \\
\hline OBC 97-C04-2 ................... & 125.872 & 21.613 & 0.4 & 0.2 & 70 & 75.2 & 8.18 & -16.69 \\
\hline 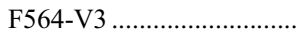 & 135.724 & 20.076 & 0.7 & 0.5 & 156 & 10.4 & 7.11 & -12.12 \\
\hline UGC $05209 \ldots \ldots \ldots \ldots \ldots \ldots \ldots$ & 146.268 & 32.238 & 0.9 & 0.9 & 0 & 11.0 & 7.30 & $\ldots$ \\
\hline 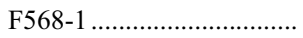 & 156.526 & 22.433 & 0.2 & 0.2 & 13 & 95.5 & 9.35 & -17.49 \\
\hline 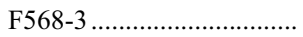 & 156.834 & 22.239 & 0.3 & 0.3 & 169 & 86.8 & 9.20 & -17.69 \\
\hline UGC $05750 \ldots \ldots \ldots \ldots \ldots \ldots$ & 158.938 & 20.990 & 1.1 & 0.6 & 167 & 62.3 & 9.00 & $\ldots$ \\
\hline 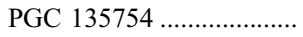 & 159.365 & 2.089 & 0.6 & 0.4 & 40 & 322.0 & 10.06 & -20.99 \\
\hline 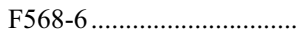 & 159.969 & 20.847 & 1.5 & 0.9 & 90 & 201.0 & 10.52 & -21.79 \\
\hline 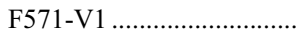 & 171.579 & 18.836 & 0.9 & 0.7 & 35 & 84.3 & 8.82 & -16.42 \\
\hline 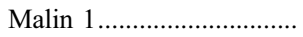 & 189.247 & 14.330 & 0.3 & 0.3 & 0 & 366.0 & 10.66 & -22.90 \\
\hline 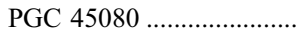 & 195.817 & 1.469 & 0.9 & 0.2 & 84 & 178.0 & 9.99 & -18.65 \\
\hline F530-1 ................................. & 316.887 & 26.450 & 0.5 & 0.3 & 52 & 199.0 & 10.27 & -20.11 \\
\hline F533-3 ............................ & 334.305 & 25.213 & 0.9 & 0.6 & 165 & 174.0 & 10.24 & -20.44 \\
\hline 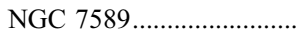 & 349.565 & 0.261 & 1.1 & 0.7 & -60 & 120.0 & 10.01 & -21.90 \\
\hline 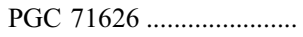 & 352.635 & -2.463 & 1.9 & 1.3 & 65 & 136.0 & 10.23 & -21.08 \\
\hline
\end{tabular}

NoтE-These properties were taken from the NED database (major and minor diameters $2 \mathrm{a}$ and $2 \mathrm{~b}$, distances) and the literature (see text).

observations (described in $\S 2$ ), including the study of the spatial distribution of the UV light $(\S 3)$ and the determination of star formation rates and efficiencies in LSBs $(\S 4)$. In $\S 5$, we discuss the color of our LSB galaxies, followed $(\S 6)$ by the consequences for the validity and interpretation of the star formation rates that we derived and the star formation history of such galaxies. This discussion may apply to other cases than LSB galaxies, such as for instance XUV-disk galaxies, considering the similarities they share.

\section{DESCRIPTION OF OUR SAMPLE AND DATA}

\subsection{LSB Sample}

In order to study the transformation of gas to stars in LSB galaxies, we compiled a list of such galaxies with a measured H I mass (taken from van der Hulst et al. 1993; de Blok et al. 1996; Matthews et al. 2001; Chung et al. 2002). We obtained GALEX Guest Investigator imaging (proposal 67, cycle 1) in order to determine the UV emission of 10 of these objects, being "massive" or giant LSB galaxies (e.g., Matthews et al. 2001). We present in this paper the observations actually performed with GALEX to date for this proposal. We also include in our analysis other LSB galaxies with $\mathrm{H}$ I measurements that were also observed by GALEX in the framework of various surveys, and for which the UV data are publicly available. Although the sample is not complete in any sense, it includes 18 galaxies ranging from dwarf to giant LSB galaxies $\left(-12.12>M_{B}>-22.90\right.$ and $\left.7.11<\log M\left(\mathrm{H}_{\mathrm{I}}\right)<10.66\right)$. Table 1 gives the position and basic information for our galaxies, taken from the NED database and the literature. The positions and position angles of a few galaxies were changed to match our GALEX imaging data.

\subsection{UV Observations and Photometry}

We have NUV imaging data for all galaxies. FUV data is available for all but three objects. Five LSB galaxies were observed in the framework of the shallow GALEX All sky Imaging Survey ( $\sim 100$ s of exposure time). We have deeper images for the other galaxies, with exposure times ranging between $\sim 1500$ and $9000 \mathrm{~s}$, depending on the survey/program (Nearby
Galaxy Survey, Guest Investigator program). The exposure time for each observation is given in Table 2. The GALEX images can be seen in Figure Set 9 of the Appendix.

$G A L E X$ photometry was performed using the same code as for the GALEXUltraviolet Atlas of Nearby Galaxies (Gil de Paz et al. 2007b), and our results are given in Table 2. For each of the two GALEX bands, we provide the magnitude measured within the last isophote that could be measured in the FUV ${ }^{8}$ ("Aperture Magnitude" in Table 2), and an asymptotic magnitude, obtained by extrapolation of the curve of growth (see details in Gil de Paz et al. 2007b). In a few cases, the uncertainties were too large to actually perform this extrapolation (i.e., the FUV magnitude of UGC 00568 and F564-V3 and the NUV magnitude of OBC 97C04-2), and we give instead the magnitude measured in the largest possible radius. Due to the faint nature of our objects, the asymptotic magnitudes suffer relatively large errors. Unless stated otherwise, we thus use the aperture magnitudes.

\subsection{Ancillary Data}

Of our 18 galaxies, 13 have been covered by the Sloan Digital Sky Survey (SDSS, DR5). For these, we downloaded from the SDSS Skyserver ${ }^{9}$ the images in the five SDSS bands, and performed the same surface photometry as on the GALEX images. In Figure Set 9, we show the $g$ band image ( $g$ and $r$ images are reasonably deep, but our objects are faint in other bands). We computed the integrated magnitude within the last radius for which the FUV flux was measured, or in NUV for galaxies without FUV data ("Last Radius" in Table 2). The SDSS images are rather shallow, especially for LSB galaxies. As a result, our integrated magnitudes have quite large error bars. On the other hand, we computed them in a similar way as the GALEX ones, and within the same apertures.

We also included in Figure Set 9 a few other magnitudes at various wavelengths: first, the $2 \mathrm{MASS} J, H$, and $K$ total magnitudes, as given in the NED database for 10 galaxies. Our study

\footnotetext{
8 The radius of this isophote corresponds to the position where the error in the azimuthally averaged surface brightness becomes larger than 0.8 mag.

9 See http://cas.sdss.org/astro/en/.
} 
TABLE 2

UV Properties of Our LSB Galaxies

\begin{tabular}{|c|c|c|c|c|c|c|c|}
\hline \multirow[b]{2}{*}{ NAME } & \multirow[b]{2}{*}{$\begin{array}{c}\text { LAST } \\
\text { Radius } \\
(\operatorname{arcsec})\end{array}$} & \multicolumn{3}{|c|}{ FUV } & \multicolumn{3}{|c|}{ NUV } \\
\hline & & $\begin{array}{l}\text { Aperture } \\
\text { Magnitude } \\
\text { (AB mag) }\end{array}$ & $\begin{array}{l}\text { Asymptotic } \\
\text { Magnitude } \\
\text { (AB mag) }\end{array}$ & $\begin{array}{c}\text { Exposure } \\
\text { Time } \\
\text { (s) }\end{array}$ & $\begin{array}{l}\text { Aperture } \\
\text { Magnitude } \\
\text { (AB mag) }\end{array}$ & $\begin{array}{l}\text { Asymptotic } \\
\text { Magnitude } \\
\text { (AB mag) }\end{array}$ & $\begin{array}{l}\text { Exposure } \\
\text { Time } \\
\text { (s) }\end{array}$ \\
\hline UGC $00568 \ldots \ldots \ldots \ldots \ldots$ & 5.00 & $22.81 \pm 0.18$ & $22.40 \pm 0.17$ & 3024.00 & $20.68 \pm 0.06$ & $18.96 \pm 0.18$ & 3024.00 \\
\hline 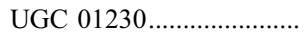 & 72.20 & $16.62 \pm 0.14$ & $16.78 \pm 0.30$ & 85.00 & $16.30 \pm 0.09$ & $16.16 \pm 0.07$ & 85.00 \\
\hline 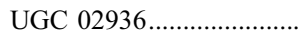 & 57.10 & $\ldots$ & $\ldots$ & $\ldots$ & $15.70 \pm 0.09$ & $15.86 \pm 0.03$ & 3382.45 \\
\hline OBC 97-C04-2.................. & 8.50 & $20.68 \pm 0.38$ & $19.44 \pm 0.66$ & 116.00 & $19.84 \pm 0.17$ & $19.52 \pm 0.18$ & 116.00 \\
\hline F564-V3 ................................ & 20.30 & $19.28 \pm 0.19$ & $19.37 \pm 0.78$ & 111.00 & $19.18 \pm 0.16$ & $18.46 \pm 0.30$ & 111.00 \\
\hline 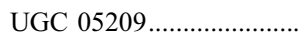 & 24.00 & $18.15 \pm 0.09$ & $18.02 \pm 0.11$ & 112.00 & $17.71 \pm 0.05$ & $17.52 \pm 0.04$ & 112.00 \\
\hline F568-1 .................................. & 36.00 & $18.16 \pm 0.03$ & $18.15 \pm 0.03$ & 1494.00 & $17.76 \pm 0.02$ & $17.74 \pm 0.02$ & 1494.00 \\
\hline F568-3 & 36.00 & $17.80 \pm 0.03$ & $17.73 \pm 0.05$ & 1494.00 & $17.49 \pm 0.02$ & $17.41 \pm 0.03$ & 1494.00 \\
\hline UGC $05750 \ldots \ldots \ldots$ & 44.30 & $17.47 \pm 0.01$ & $17.59 \pm 0.01$ & 3530.00 & $17.09 \pm 0.01$ & $17.17 \pm 0.01$ & 3531.00 \\
\hline PGC 135754 & 24.50 & $19.75 \pm 0.05$ & $19.54 \pm 0.10$ & 3164.00 & $18.95 \pm 0.03$ & $18.70 \pm 0.02$ & 3164.00 \\
\hline F568-6 ............................ & 120.80 & $\ldots$ & & 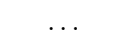 & $16.89 \pm 0.21$ & $16.87 \pm 0.01$ & 3573.00 \\
\hline F571-V1 ................................. & 21.20 & $19.01 \pm 0.14$ & $18.47 \pm 0.35$ & 109.00 & $18.51 \pm 0.08$ & $18.14 \pm 0.12$ & 109.00 \\
\hline 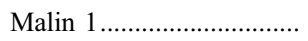 & 48.00 & $19.61 \pm 0.09$ & $19.52 \pm 0.45$ & 1841.50 & $18.77 \pm 0.05$ & $18.30 \pm 0.08$ & 1841.50 \\
\hline 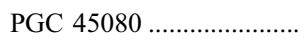 & 48.10 & $\ldots$ & & $\ldots$ & $17.77 \pm 0.02$ & $17.50 \pm 0.02$ & 1377.00 \\
\hline 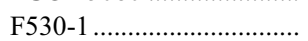 & 27.90 & $18.26 \pm 0.08$ & $18.12 \pm 0.05$ & 3392.85 & $17.53 \pm 0.02$ & $17.29 \pm 0.01$ & 9040.00 \\
\hline F533-3 …....................... & 49.00 & $18.11 \pm 0.03$ & $18.07 \pm 0.04$ & 4317.90 & $17.55 \pm 0.01$ & $17.45 \pm 0.01$ & 5924.05 \\
\hline 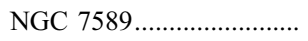 & 38.30 & $17.70 \pm 0.03$ & $17.53 \pm 0.02$ & 1495.00 & $17.21 \pm 0.01$ & $17.05 \pm 0.02$ & 1495.00 \\
\hline 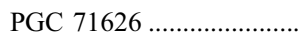 & 84.40 & $17.05 \pm 0.09$ & $16.96 \pm 0.03$ & 3411.00 & $16.33 \pm 0.01$ & $16.21 \pm 0.05$ & 3411.00 \\
\hline
\end{tabular}

NoтE-UV photometry: last radius measured, aperture magnitude within the last radius, asymptotic magnitude, and exposure time for the FUV and NUV bands of GALEX (see Gil de Paz et al. 2007b for details on these values and how they are obtained).

overlaps with a few works on LSB galaxies, from which we also took total magnitudes, as published: de Blok et al. (1995) for F564-V3, F568-1, F568-3, F571-V1, UGC 01230, UGC 05209, and UGC 05750; McGaugh \& Bothun (1994) for UGC 01230 and F568-6; and Hunter \& Elmegreen (2006) for F564-V3 and UGC 05209.

While we performed the SDSS photometry following the same procedures as for the UV, these other studies are independent, and some differences might exist in, e.g., the position, position angle, and aperture. Nevertheless, they give an idea of the overall shape of the galaxy SED when compared to other magnitudes, as is done in Figure Set 9.

\subsection{Reference Samples}

In order to compare our results to high surface brightness galaxies, we considered two large samples typical of "normal" (non-LSB) star-forming galaxies.

The first one is the GALEX Atlas of nearby galaxies (Gil de Paz et al. 2007b). The big advantage of this sample is that the NUV and FUV photometry were performed in the same way and with the same code as for our galaxies. Galaxies in the Atlas are not selected on the basis of their surface brightness, but are representative of nearby galaxies. Their properties are actually consistent with the Nearby Field Galaxy Survey of Jansen et al. (2000). Although some LSB (or intermediate surface brightness) galaxies might be included, the typical star-forming galaxies in the Atlas are high surface brightness objects. For this sample, we queried the LEDA database (Paturel et al. 2003) to obtain H I magnitudes and converted them into $\mathrm{H}$ I masses.

The second sample is a set of star-forming galaxies from the GOLD Mine database (Gavazzi et al. 2003), including multiwavelength data for a large number of cluster galaxies. The advantage of this sample is that it includes a determination of the $\mathrm{H}$ I deficiency. The $\mathrm{H}$ I deficiency is defined as the logarithmic difference between the average $\mathrm{H}$ I mass of a reference sample of isolated galaxies of similar type and linear dimension and the $\mathrm{H}$ I mass actually observed in individual objects (Gavazzi et al. 2005). We excluded all galaxies with $\mathrm{H}_{\mathrm{I}}$ deficiencies larger than 0.3 , which is typically found in perturbed galaxies (e.g., ram-pressure stripping within clusters). For this sample, the attenuation in the UV was already estimated (following the method of Boselli et al. 2003), so the FUV and NUV magnitudes can be corrected for internal extinction.

\section{SPATIAL EXTENT OF THE UV EMISSION}

\subsection{Extended Emission}

We inspected our images to determine which of our galaxies present a XUV-disk-like morphology, except for the five galaxies from the All-Sky Imaging Survey (AIS) which are too shallow to really discuss this point.

Thilker et al. (2007) defined two types of XUV-disk galaxies. Type 1 XUV-disk galaxies present structured UV-bright emission complexes beyond the anticipated location of the star formation threshold (corresponding to a NUV surface brightness of about 27.35 $\mathrm{AB}$ mag $\operatorname{arsec}^{-2}$ ). Six of our galaxies correspond to this case: NGC 7589, PGC 135754, PGC 71626, F533-3, Malin 1, and F568-6. Type 2 XUV-disk galaxies are defined by Thilker et al. (2007) as galaxies forming stars (i.e., UV-bright) over an area much larger than the spatial extent of their old stellar population (as traced by near-infrared light). Although we do not have deep enough $K$-band images to use their quantitative definition, the comparison of UV and optical images strongly suggests that F568-1, F530-1, and F568-3 are actually type 2 XUV-disk galaxies (we thus find the same fraction of type 1 vs. type 2 as Thilker et al. [2007], 2 for 1, even if we have a very small number of objects).

Thilker et al. (2007) found that about $30 \%$ of the galaxies in the GALEX Atlas of nearby galaxies fall into any of the XUV categories, and Zaritsky \& Christlein (2007) found at the 90\% confidence level that $27 \%$ of the spirals have UV sources in their disks at radius between 1.25 and 2 optical radii, showing that extended 
UV emission is common in nearby spirals. Out of 13 deep UV images of LSB galaxies, 9 present clear signs of extended emission; thus, this phenomenon is even more frequent in LSB than normal galaxies, although we might be suffering from poor statistics.

In the following, we will discuss the UV light distribution in a few galaxies of special interest. Recent studies suggested that Malin 1 is an early-type galaxy surrounded by a huge LSB disk (Sancisi \& Fraternali 2007; Barth 2007). The UV images of Malin 1 show the central part of the galaxy very clearly. In the outer disk, we observe several diffuse emission regions, probably corresponding to relatively recent star formation within the LSB disk (see also Thilker et al. 2007). Malin 2 (F568-6) presents a spectacular extended UV disk with a clear spiral morphology, while it can only be guessed in optical (DSS or SDSS images). Spiral patterns at large radii are observed in several of our other galaxies (PGC 71626, NGC 7589, F530-3). They are often barely visible in the optical, but the contrast is much more favorable at the UV wavelengths. This is due to the fact that the arm-interarm contrast is much more favorable in the UV if star formation is enhanced in the arms (UV-emitting stars have short lifetime, and thus are found closer to their formation locus), and owing to the low background in the UV. Some other galaxies may harbor star formation within spiral patterns that cannot be seen in our images because of short exposure times or low spatial resolution (e.g., F568-3, F568-1).

\subsection{Optical to UV Size Ratio}

Figure 1 shows that while our LSB galaxies cover the range of optical diameters found in the GALEX Atlas of Nearby Galaxies (Gil de Paz et al. 2007b), the optical to UV diameter ratio in LSB galaxies is on average much smaller (by about a factor 2) than the same quantity for the galaxies in the Atlas, considering either the last UV isophote (top) or the diameter in which $80 \%$ of the UV flux is contained. Thus, the UV light in LSB galaxies is on average more extended with respect to the optical than "normal" galaxies.

The diameter corresponding to the last radius where the UV isophote was computed (top panel of Fig. 1) corresponds to a limiting signal-to-noise level, sensitive to the depth of the images. Comparison to the one measured in the GALEX Atlas of nearby galaxies makes sense for most galaxies, since the exposure times are of the same order of magnitude (within a factor of a few). We can thus make a quantitative comparison of this diameter ratio in our LSB galaxies and in the GALEX Atlas. This is not the case, however, for the data taken from the All-Sky Imaging Survey. For them, if we had deeper images (similar to the one in the Atlas), we could measure UV isophotes farther away from the center than we actually did, and we would find a lower optical to UV ratio. This effect, indicated by arrows in Figure 1, can thus only strengthen our conclusion.

The fact that the optical/UV diameter ratio of LSB galaxies is low may be a sign that these galaxies are relatively unevolved objects, having formed stars in the past only in their central part, with large-scale star formation occurring currently in a large $\mathrm{H}$ I disk. This is consistent with the analysis of Bell et al. (2000), who found optical-near-infrared color gradients showing younger ages in the outer parts of LSB galaxies.

\section{STAR FORMATION RATES AND STAR FORMATION EFFICIENCY IN LSB GALAXIES}

\subsection{Theoretical Expectations}

The models for LSB and HSB galaxies of Boissier et al. (2003) and Boissier \& Prantzos (2000) predict that the lower

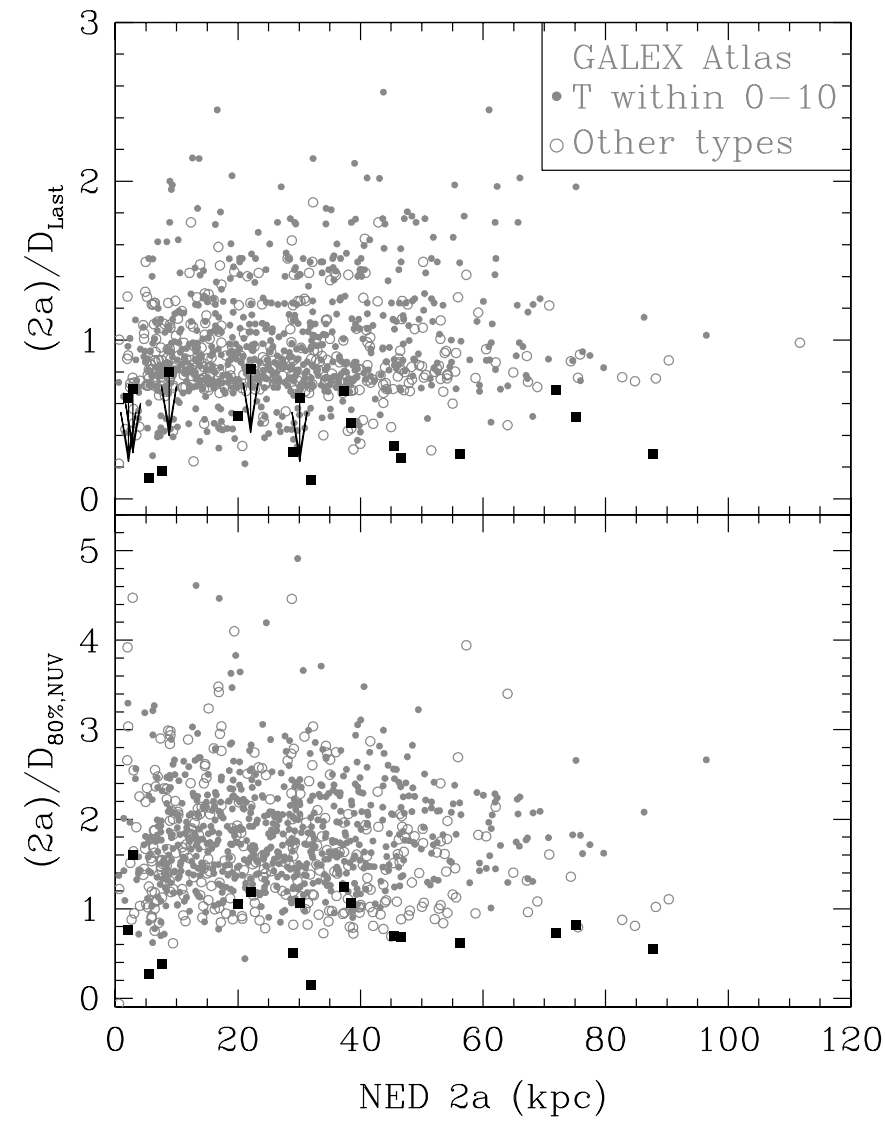

FIG. 1.- Ratio of the optical to the total NUV diameter (top) and $80 \%$ (bottom) NUV diameter (diameter including $80 \%$ of the light) as a function of the optical diameter of the galaxies in our sample (squares). Open and filled circles are the same quantities for the GALEX Atlas of Nearby Galaxies (Gil de Paz et al. 2007b). The last radius depending on the exposure time, galaxies from the All Sky Imaging Survey would have larger UV diameter (and lower optical to UV diameter ratio) if observed at the depth of the GALEX Atlas. In that case, the ratio would be smaller than the one we determined, which we indicated in the top panel with arrows. [See the electronic edition of the Journal for a color version of this figure.]

surface density and larger radial extent in massive LSB objects make them less efficient at forming stars by a factor of 5 to 10 . These models are of the general type that consider LSB galaxies as analogs to HSB galaxies, but with larger specific angular momentum (Jimenez et al. 1998; Dalcanton et al. 1997). For the same total mass, the surface densities are lower. If the star formation density is not a linear function of the gas density, a lower star formation efficiency is to be expected. This is the case in the models of Boissier et al. (2003), where the star formation density $\left(\Sigma_{\mathrm{SFR}}\right)$ is proportional to $\Sigma_{\text {gas }}$ to a power of 1.5 , and to the inverse of the radius. It is also the case in the models of Jimenez et al. (1998), who assume $\Sigma_{\text {SFR }} \propto \Sigma_{\text {gas }}^{1.5} \Sigma_{\text {stars }}^{0.5}$. Indeed, in both cases the ratio $\Sigma_{\mathrm{SFR}} / \Sigma_{\text {gas }}$ will be smaller for larger, lower density LSB galaxies. De Blok et al. (1996) and McGaugh (1992) showed that the gas surface densities in LSB galaxies are lower by a smaller fraction than the stellar surface densities when compared to HSB galaxies (a factor of 5 or 10 in surface brightness, corresponding to only $2-3$ in gas density). This result is qualitatively consistent with such a star formation law: for instance, considering the star formation law used by Boissier et al. (2003) we have

$$
\frac{\Sigma_{\mathrm{SFR}, \mathrm{HSB}}}{\Sigma_{\mathrm{SSFR}, \mathrm{LSB}}}=\left(\frac{\Sigma_{\mathrm{gas}, \mathrm{HSB}}}{\Sigma_{\mathrm{gas}, \mathrm{LSB}}}\right)^{1.5} \frac{R_{\mathrm{LSB}}}{R_{\mathrm{HSB}}},
$$


TABLE 3

SFR Deduced from the Kennicutt (1998) Conversion Factor, Compared to SFR from Other Studies

\begin{tabular}{|c|c|c|c|c|}
\hline Name & $\begin{array}{l}\text { FUV SFR } \\
\left(M_{\odot} \mathrm{yr}^{-1}\right)\end{array}$ & $\begin{array}{l}\text { NUV SFR } \\
\left(M_{\odot} \mathrm{yr}^{-1}\right)\end{array}$ & $\begin{array}{c}\text { Other } \\
\left(M_{\odot} \mathrm{yr}^{-1}\right)\end{array}$ & $\begin{array}{c}\text { Other Source, } \\
\text { Comments }\end{array}$ \\
\hline 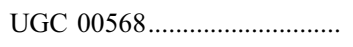 & 0.0165 & 0.1174 & & Early-type SED, measured only in the central $5^{\prime \prime}$ \\
\hline 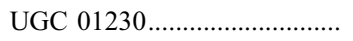 & 0.3311 & 0.4446 & & \\
\hline 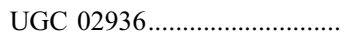 & $\ldots$ & 0.8366 & & \\
\hline 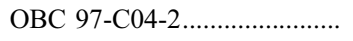 & 0.0184 & 0.0399 & & \\
\hline 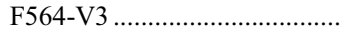 & 0.0013 & 0.0014 & & \\
\hline UGC $05209 \ldots \ldots \ldots \ldots \ldots \ldots \ldots \ldots$ & 0.0040 & 0.0061 & & \\
\hline 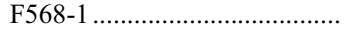 & 0.3020 & 0.4365 & 0.31 & van den Hoek et al. (2000) model \\
\hline 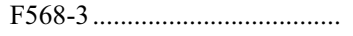 & 0.3476 & 0.4624 & 0.33 & van den Hoek et al. (2000) model \\
\hline UGC $05750 \ldots \ldots \ldots \ldots \ldots \ldots \ldots \ldots$ & 0.2426 & 0.3443 & & \\
\hline 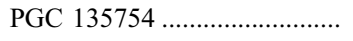 & 0.7938 & 1.6585 & & \\
\hline 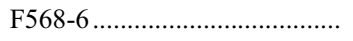 & $\ldots$ & 4.3092 & & \\
\hline 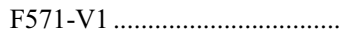 & 0.1076 & 0.1705 & 0.14 & van den Hoek et al. (2000) model \\
\hline Malin 1 .......................................... & 1.1667 & 2.5291 & 0.38 & Rahman et al. (2007) infrared \\
\hline PGC $45080 \ldots \ldots \ldots \ldots \ldots \ldots \ldots \ldots \ldots$ & & 1.5026 & & \\
\hline F530-1 …… & 1.1959 & 2.3426 & & \\
\hline F533-3 …… & 1.0498 & 1.7583 & & \\
\hline 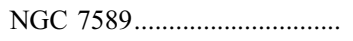 & 0.7284 & 1.1438 & & \\
\hline 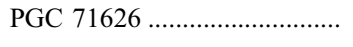 & 1.7025 & 3.3043 & & \\
\hline \multicolumn{3}{|c|}{ LSB galaxies $\left(-15.14>M_{B}>-21.17\right) \ldots \ldots$} & $\sim 0.2$ & McGaugh \& Bothun (1994) model \\
\hline \multicolumn{3}{|c|}{ 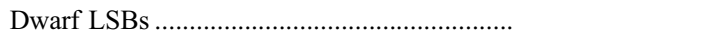 } & $0.0083-0.35$ & van Zee et al. (1997) H $\alpha$ imaging \\
\hline \multicolumn{3}{|c|}{$\begin{array}{l}\text { Subsample of APM, } \mu_{0}>21 \\
16 \text { relatively faint LSBs. }\end{array}$} & $0.17 \pm 0.36$ & Burkholder et al. (2001) $\mathrm{H} \alpha$, nuclear \\
\hline & median 0.15 & van den Hoek et al. (2000) models \\
\hline & & 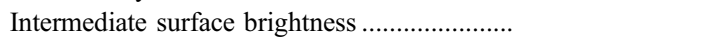 & $0.3-5$ & O’Neil et al. (2007) \\
\hline
\end{tabular}

NotE.-Caution should be taken concerning the values from other sources; see $\S 4$.

where $R_{\mathrm{LSB}}$ and $R_{\mathrm{HSB}}$ are typical sizes of LSB and HSB galaxies. Assuming that they encompass the same total $\mathrm{H}$ I mass, we can write $R_{\mathrm{LSB}(\mathrm{HSB})} \propto \Sigma_{\text {gas, } \mathrm{LSB}(\mathrm{HSB})}^{-0.5}$, and then rewrite equation (1) as $\left(\Sigma_{\mathrm{SFR}, \mathrm{HSB}} / \Sigma_{\mathrm{SFR}, \mathrm{LSB}}\right)=\left(\Sigma_{\mathrm{gas}, \mathrm{HSB}} / \Sigma_{\mathrm{gas}, \mathrm{LSB}}\right)^{1.5+0.5}$. With this simple back-of-the-envelope calculation, we find that a factor of 3 difference in the gas density can produce a factor of 9 difference in the star formation rate surface density between LSB and HSB galaxies. If the gas surface density is approximately constant during the history of the galaxy, the same factor applies to stellar surface densities.

Note that observationally, we use integrated values rather than surface densities. These are equivalent because the UV diameter (in which the UV is detected) is roughly the diameter where the gas column density reaches $1 M_{\odot} \mathrm{pc}^{-2}$, at least for those galaxies for which it has been measured ( $\mathrm{H}$ I diameters are, however, quite uncertain [Matthews et al. 2001], and thus the gas surface densities defined in that way, and the star formation law derived from them, are crudely defined).

Gerritsen \& de Blok (1999) performed $N$-body simulations in which star formation and feedback are implemented in a very different way (based on the Jeans mass). In their case, they did not find differences in the SFR with respect to the HSB case; however they still found a lower SFR for LSB galaxies when including the effects of the low metallicity in LSB galaxies, lowering the cooling efficiency and thus the amount of star formation. Based on their work, a lower efficiency should then be expected in LSB galaxies, but due to their lower metal content.

The lower global efficiency required to form stars in LSB galaxies was also expected in the models of van der Hulst et al. (1993), because their gas surface density is smaller than the "star formation threshold."

\subsection{Empirical Determinations of the SFR}

In this section, we assume the standard conversion factors from the UV to derive the star formation rate following Kennicutt
(1998). The results are given in Table 3 . We caution that the these conversions assume that the star formation rate is roughly constant over a few $10^{8} \mathrm{yr}$, that there is no attenuation by dust (or this effect has been corrected), and that the galaxy initial mass function is standard (close to Salpeter). We will see in the next sections that some of these assumptions might not be valid, which will greatly affect our result.

Our UV SFRs are larger than the ones determined in various papers concerning LSB galaxies (see Table 3 and Fig. 2). There are several reasons for this, which we discuss below. (1) In the absence (or deficiency) of dust (as seems to be the case; see $\S 4.3$ ), the infrared will provide underestimated measurements of the star formation rate (Rahman et al. 2007). (2) Some studies (Burkholder et al. 2001) provide the nuclear SFR, while we provide integrated measurements for these significantly extended galaxies. (3) The SFRs of van Zee et al. (1997) are a bit smaller than ours for the same $\mathrm{H}$ I mass (the same is true for a given $B$ magnitude). They are derived from $\mathrm{H} \alpha$ data. This could indicate an age (the most massive stars have disappeared) or an IMF (massive stars were not formed in the first place) effect. Note that their sample consists of mostly dwarf galaxies. O'Neil et al. (2007) also determined the SFR from $\mathrm{H} \alpha$, but for more massive galaxies, with intermediate surface brightness. Their results are quite similar to ours (see Fig. 2).

On the other hand, the SFRs obtained by van den Hoek et al. (2000) from modeling broadband and H I content are in good agreement with the ones in our sample at the same magnitude, or $\mathrm{H}$ I mass (one of their galaxy has a large SFR, but is uncertain due to contamination by other sources). Note that they sample relatively faint LSB galaxies and not the more massive ones for which we have more data and see more differences with respect to "normal" spirals.

In Figure 2, we compare the relation between SFR (derived from NUV) and $\mathrm{H}$ I in our sample of LSB galaxies to the one in 


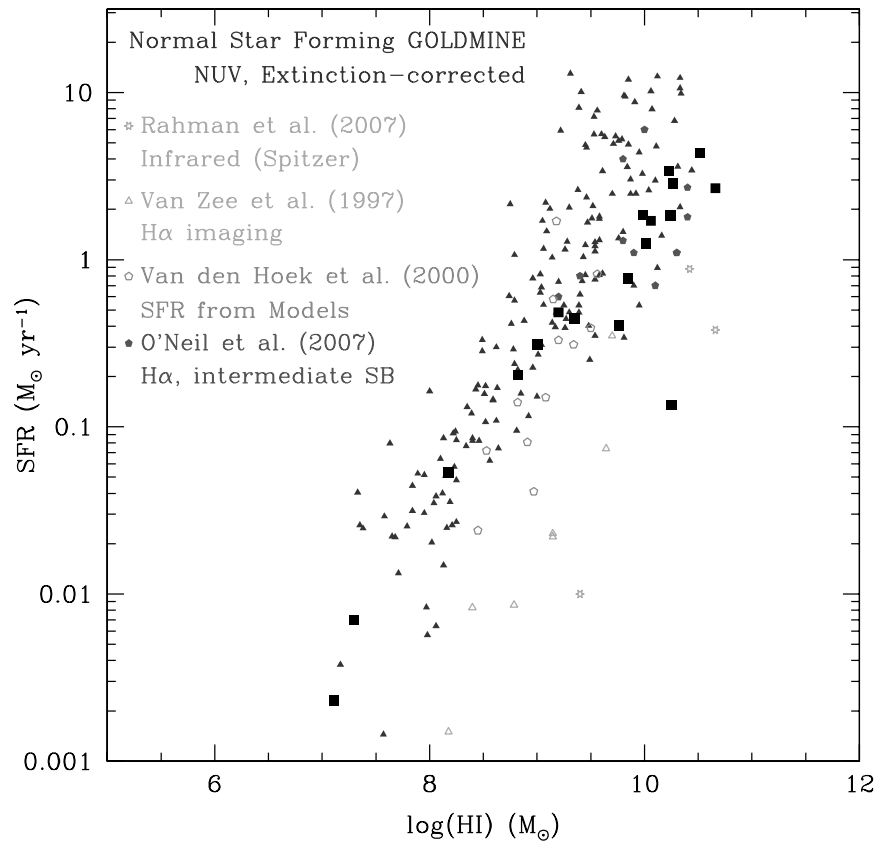

FIG. 2.-NUV-derived SFR as a function of the H I mass in LSB galaxies ( filled squares), compared to extinction-corrected SFR in normal star-forming galaxies (from GOLD Mine), and other determinations in LSB galaxies. These values are, however, very uncertain. [See the electronic edition of the Journal for a color version of this figure.]

nearby star-forming galaxies from GOLD Mine, corrected for extinction effects. We find that the SFR in the massive LSB is lower, thus the efficiency of forming stars (or at least NUVemitting stars) is lower in such galaxies (we checked that the massive GOLD Mine galaxies have still larger SFR if we use data uncorrected for extinction for them). This is in nice agreement with the theoretical expectations discussed above that the star formation efficiency should be lower (see, however, the next sections, in which we show that determining SFR in LSB galaxies may be a harder task than is done here and in most empirical works). The few low-mass LSB galaxies we have in our sample seem similar to "normal" star-forming galaxies for their mass, in terms of star formation rate.

\section{FUV-NUV COLOR OF LSB GALAXIES}

\subsection{Observations}

We checked that the FUV-NUV color profiles of our galaxies (not shown) are quite flat, so that the integrated color is similar to the color all over the disk, including the outer regions. UGC 00568 is an exception: this galaxy is barely visible in the FUV image, and it was not possible to extract a reliable profile in this band. As a result, the FUV-NUV color corresponds only to the central $7^{\prime \prime}$. The rest of the disk is probably also red, since it is not detected in the FUV band. This is the only object in our sample that shows a SED similar to those of early-type galaxies (see Fig. Set 9 in the Appendix). Note that even if the magnitudes are integrated within a larger radius, the NUV-2MASS colors are still consistent with an early-type galaxy SED. This is our only case of an extremely red LSB despite a huge $\mathrm{H}$ I reservoir.

In normal star-forming galaxies, the total infrared (TIR) to UV emission ratio of a galaxy is a good proxy for the attenuation (Buat \& Xu 1996; Gordon et al. 2000; Panuzzo et al. 2003). Several studies (Boissier et al. 2007; Gil de Paz et al. 2007b; Cortese et al. 2006; Seibert et al. 2005) have shown that a relation exists between this ratio and the FUV-NUV color in star-

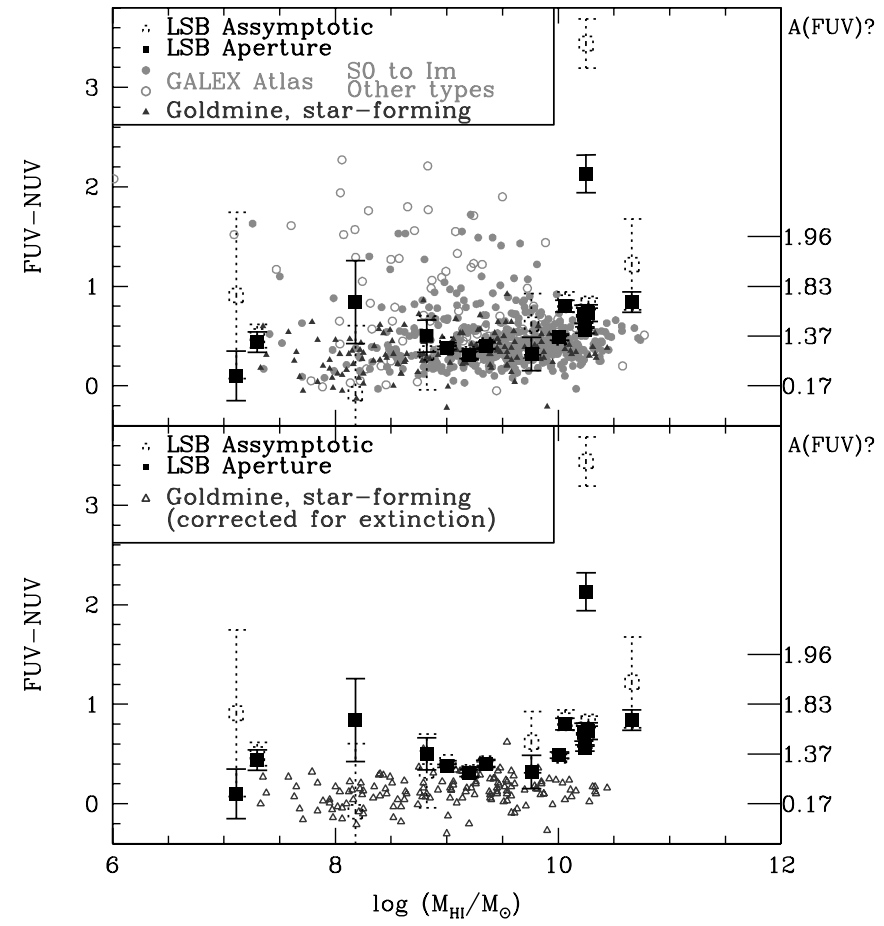

FIG. 3.- Observed FUV-NUV color of our sample of LSB galaxies, using aperture (squares) or asymptotic (open circles) magnitudes, as a function of the $\mathrm{H}$ I mass, compared to a sample of normal star-forming galaxies from GOLD Mine, and to the GALEX Atlas of Nearby Galaxies. In the top panel, the LSB color is compared to the FUV-NUV color observed in various samples. In the bottom panel they are compared to the FUV-NUV color corrected for extinction in the GOLD Mine sample. The right axis indicates the amount of extinction derived using the relationship between the infrared to UV ratio and FUV-NUV in Boissier et al. (2007) and the $A$ (FUV) vs. infrared to UV ratio in Buat et al. (2005). These fits are only valid for FUV $-\mathrm{NUV} \lesssim 1.5$. We included a question mark in the label $A(\mathrm{FUV})$ to stress that this is the extinction one would derive from the color for usual galaxies. However, this conversion may not be valid for LSB galaxies (see text of $\S 5$ ). [See the electronic edition of the Journal for a color version of this figure.]

forming galaxies, even if it is shifted (toward lower extinction for the same color) with respect to the classical relationship found with IUE in starbursts (e.g., Heckman et al. 1995; Meurer et al. 1995, 1999). Thus, it is expected that star-forming galaxies with low extinction should have blue FUV-NUV colors close to zero, while redder colors should indicate some amount of extinction.

The FUV-NUV colors of our LSB galaxies are similar to the ones measured in normal star-forming galaxies, and follow similar trends with various quantities (we show in Fig. 3 the FUV-NUV color vs. the H I mass). From this figure, it even seems that for the same mass, the color is marginally on the red side of normal galaxies, especially for LSB galaxies with $\mathrm{H} \mathrm{I}$ masses above $10^{10} M_{\odot}$. The bottom panel of Figure 3 clearly shows that LSB galaxies are almost all redder than the FUVNUV color of star-forming galaxies when they are corrected for attenuation. Such corrections are uncertain, but the corrected colors do correspond to what is expected in the stellar populations of star-forming galaxies ( FUV-NUV $\sim 0$; see $\S 5.3$ ). A natural explanation of the FUV-NUV color of LSB galaxies is thus that they suffer large internal attenuation, similar to HSB normal starforming galaxies. We combined the Buat et al. (2005) relationship (FUV attenuation as a function of the TIR/FUV ratio) and the Boissier et al. (2007) relationship (TIR/FUV as a function of FUV-NUV in nearby spirals) in order to convert the FUVNUV color into the corresponding amount of extinction in normal galaxies. An extinction scale computed in that way is shown 

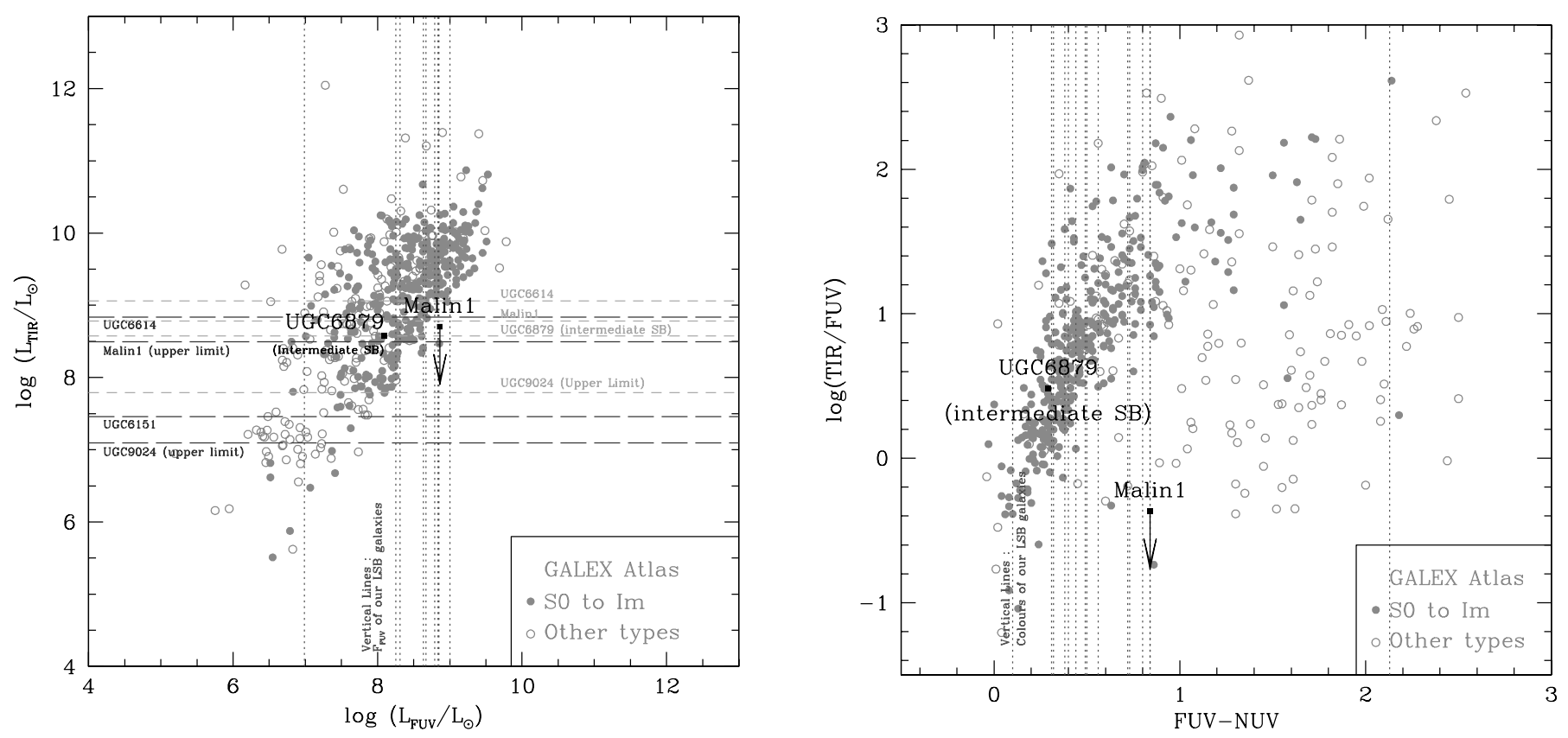

Fig. 4.-Left: FUV vs. TIR luminosities. The horizontal (vertical) lines indicate TIR (FUV) values for galaxies in Rahman et al. (2007) and Hinz et al. (2007) (our work only showing galaxies in the same $M_{B}$ magnitude range as these studies). Right: TIR to FUV ratio vs. FUV-NUV color. [See the electronic edition of the Journal for a color version of this figure.]

in the right axis of Figure 3 (we cannot directly use the TIR/FUV ratio, since we do not have infrared measurements for the galaxies in our sample, with the exception of Malin 1; see $\S$ 5.2). This gives us the amount of attenuation $A$ (FUV) necessary to redden the FUV-NUV color to the observed level, about $1.5 \mathrm{mag}$ for the massive LSB galaxies. We study this assumption in $\S 5.2$.

We emphasize that this calibration of the attenuation with the FUV - NUV ratio should be valid only for star-forming galaxies; if star formation were quenched some time ago (early type, truncated SFR), this relation will fail (e.g., Boselli et al. 2006; Cortese et al. 2008) because old stars would have a red FUVNUV color even in the absence of dust. However, our SEDs are consistent with those of irregular and late-type spirals, in which such quenching should not a priori occur, except for UGC 00568. A few other galaxies have not such late type (similar colors to the Sbc template), but they are not systematically the redder ones in FUV-NUV. We note also that optical/red images show a more concentrated morphology than the UV, i.e., the color of star-forming regions, especially outer ones, are bluer than the integrated one (for the galaxies with SDSS data, we verified this point with the NUV $-r$ color profile). This is an indication that the FUV-NUV and, e.g., NUV $-r$ color do not trace the same stellar population. In this case the FUV-NUV red color could correspond to star formation that was quenched recently in the young regions, while the optical colors would be more sensitive to a smooth star formation history on the timescales corresponding to older populations. Alternatively, redder FUV -NUV colors could also be explained by an IMF effect (we will come back to such scenarios in $\S 5.3$ ).

\subsection{Are LSB Galaxies Affected by Dust Attenuation?}

As discussed above, the FUV-NUV color in our LSB galaxies could indicate significant amount of attenuation, increasing with the $\mathrm{H}$ I mass of galaxies. However, it is generally believed that LSB disks are deficient in dust with respect to their HSB counterparts (e.g., Rahman et al. 2007 and references therein), based on their generally blue colors, low densities, low metallicities, and deficiency in molecular gas. Actually, recent studies show that LSB galaxies do contain molecular gas (although in smaller amount with respect to HSB disks), which, being localized in isolated regions, is difficult to detect (O'Neil \& Schinnerer 2004; Das et al. 2006). We should also note that measuring the $\mathrm{CO}$ molecular emission and converting it to gas mass is quite uncertain due to the uncertainty in the conversion factor from $\mathrm{CO}$ to $\mathrm{H}_{2}$ (Boselli et al. 2002), especially at the low metal abundances and densities found in these galaxies. Among the reasons for thinking that the attenuation in LSB galaxies is weak, we should also note that LSB disks are found to be transparent by Holwerda et al. (2005) based on the count of distant field galaxies seen through disks.

Very recently, it became possible to study the far-infrared dust emission in LSB galaxies with the Spitzer Space Telescope. Hinz et al. (2007) reported observations of five LSB galaxies with Spitzer, three of them also analyzed (with slightly different results) in Rahman et al. (2007), together with one "intermediate" surface brightness galaxy. Hinz et al. (2007) concluded that the far-infrared emission is weak when compared to normal starforming galaxies, and that LSB galaxies contain less and/or colder dust. They suggested that the dust is detected in the infrared for galaxies with large amounts of star formation. From the MIPS data in these papers, we computed the total infrared (TIR) emission (using eq. [4] of Dale \& Helou 2002) and show them as horizontal dashed lines in the left panel of Figure 4. The vertical dotted lines indicates FUV fluxes for LSB galaxies in our sample with blue band magnitudes in the same range $\left(-23<M_{B}<\right.$ -17) as the galaxies in Rahman et al. (2007) and Hinz et al. (2007). Assuming that LSB galaxies with similar $M_{B}$ are indeed similar, LSB galaxies should lie in this diagram in the region where the horizontal and vertical lines cross (note that many horizontal lines are actually upper limits). The TIR emission of the galaxies in the GALEX Atlas of Nearby Galaxies (computed from IRAS 60 and $100 \mu \mathrm{m}$ fluxes as in Dale et al. 2001) is also 


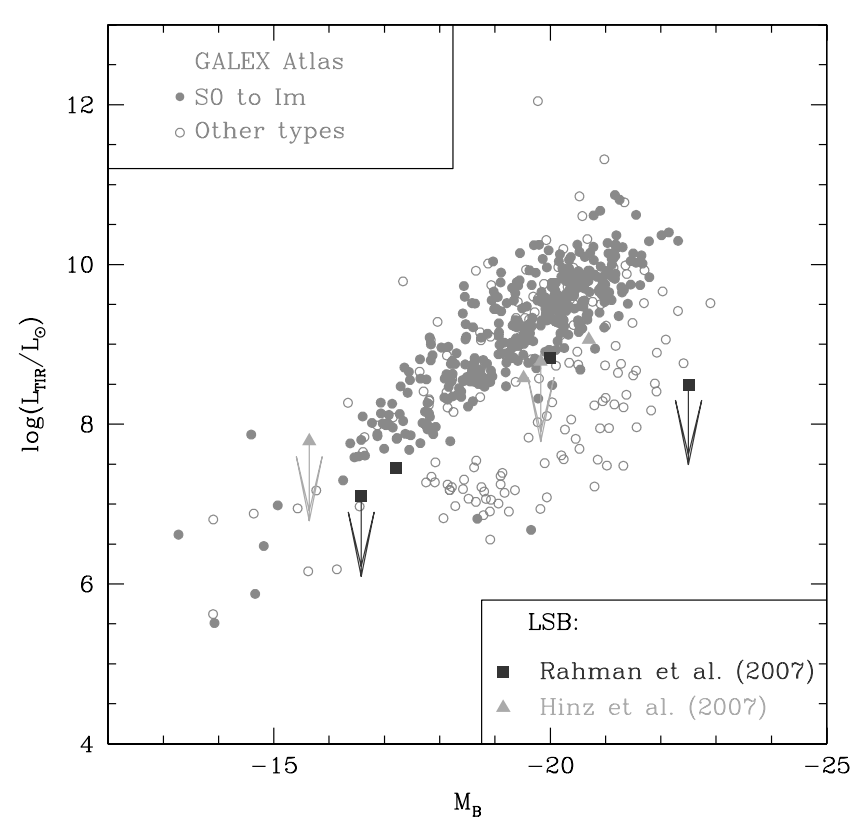

FIG. 5.- TIR luminosities as a function of the blue band absolute magnitude for galaxies of the GALEX Atlas of nearby galaxies (circles) and for LSB galaxies (squares and triangles). [See the electronic edition of the Journal for a color version of this figure.]

shown. Measured TIR values in massive LSB galaxies are clearly weaker that the values found in normal star-forming galaxies with the same FUV flux. Malin 1 is the only true LSB galaxies for which we have both TIR and FUV values, and that can be definitively placed in this diagram. We find that its TIR flux (actually an upper limit) is smaller than for any normal star-forming galaxies with the same FUV flux. We note that UGC 06879 is considered by Rahman et al. (2007) as an intermediate surface brightness galaxy. Using the UV fluxes from Gil de Paz et al. (2007b), we see that it is located among normal star forming galaxies in this diagram.

The right panel of Figure 4 shows the ratio TIR/FUV vs. the FUV-NUV color. We can see the trend followed by normal starforming galaxies mentioned above; redder galaxies are more extinguished. The intermediate surface brightness galaxy from Rahman et al. (2007) is on this line. The upper limit on the farinfrared emission of Malin 1 from Rahman et al. (2007) corresponds to a very low upper limit on this ratio, resulting in an upper limit of 0.4 mag of attenuation with the calibration of Buat et al. (2005). Hinz et al. (2007) obtained lower numbers for the infrared fluxes upper limits of Malin 1, stressing the difficulty of determining them in LSB galaxies. Adopting their results, we obtain an even lower value for the maximal FUV attenuation in Malin 1, of $0.1 \mathrm{mag}$ (similar attenuations of 0.39 and $0.2 \mathrm{mag}$ are obtained using the calibration of Cortese et al. [2008], based on the FUV - g color of Malin 1). In any case, this is much lower than the attenuation necessary to redden the FUV-NUV color estimated in $\S 5.1(\sim 1.5 \mathrm{mag})$.

Unfortunately, at present we have both FUV and TIR measurements only for Malin 1. Instead of comparing the TIR and FUV emission of galaxies with similar blue band magnitude $M_{B}$, as we did above, we can also directly compare the relationship between the TIR emission and $M_{B}$ for the few LSB galaxies for which we have this information to the one obtained with the GALEX Atlas of nearby galaxies (Fig. 5). Here again, we see that LSB galaxies have systematically low dust emission in the infrared with respect to normal star-forming galaxies with the same $M_{B}$. This suggests that they suffer a lower amount of extinction.

Putting together the results from this section, although the trends followed by the FUV-NUV color in LSB galaxies are similar to the ones observed in HSB galaxies, which are due (we believe) to an attenuation effect, other considerations (especially infrared fluxes) clearly suggest that there is very little extinction even in the more massive, redder LSB galaxies. In the following, we seek for other explanation for their FUV-NUV color.

\subsection{Age or IMF Effect?}

Figure 4 shows that Malin 1 (and probably other massive LSB disks) have a low value of TIR/FUV for a large value of FUV-NUV, with respect to "normal" (non-LSB) star forming galaxies. Kong et al. (2004), for instance, used models to show that the position of a galaxy in this diagram may depend on its star formation history. In order to match the position of Malin 1 with their models, it would be necessary to assume extremely low current-to-past average star formation rate (birthrate $b$ parameter). This is, however, not very compatible with the idea that LSB galaxies are "young," i.e., to the light of their stellar population and chemical state (large gas fraction, low metallicity, blue optical colors in many galaxies). The spectrum resulting from such a history should be similar to early-type galaxies. Only one galaxy in our sample has such a spectrum (UGC 00568); the remaining galaxies are indeed similar to star-forming irregular and late spirals types. One possible explanation would be that star formation proceed by bursts with quiescent phases longer than the UV emission timescale (a few hundred million years), but shorter than the optical emission timescale (Gyr scale). During quiescent phases this could produce red FUV-NUV colors, relatively blue optical colors, and low birthrate parameters (UGC 00568 would be an extreme case in which the quiescent phase was long enough to also affect optical colors).

Indeed, in the absence of extinction, the FUV-NUV color can be a good indicator of the age of a star cluster (Bianchi et al. 2005). FUV-NUV color ranging from about -0.2 to 1 would correspond roughly to ages of the clusters within $3 \mathrm{Myr}$ to $360 \mathrm{Myr}$ according to this paper. If the FUV-NUV color results from such an age effect, its dependence on the $B$-band magnitude or $\mathrm{H}$ I mass (redder colors are found in the most massive LSB disks) still remains to be explained, since it is not obvious why the age of the most recent star formation event should behave in this way. Part of the trend could actually be due to a metallicity effect, since more massive galaxies are more metal-rich (see $\S 6$ for a detailed derivation of this age, taking into account the metallicity).

An alternative would be that the IMF is different in lowdensity regions, favoring lower mass stars, which are redder. Indeed, Weidner \& Kroupa (2005) found that the "integrated Galaxial" IMF is steeper than the universal IMF, assuming stars are born in clusters following a mass function whose the maximum mass $\left(M_{\mathrm{ecl} \text { max }}\right)$ is a function of the galaxy SFR (stars are born following the IMF within these clusters). They predict a strong effect at low star formation rates, and predict that stars more massive than $25 M_{\odot}$ will never form in low-mass LSB galaxies. When the IMF gets steeper, favoring less massive stars, we should obtain redder colors. Moreover, with such an effect, we should find that less massive galaxies, in which lower amount of star formation takes place, should be redder, while we observe the opposite trend (see Fig. 3 for FUV-NUV vs. the H I mass; a similar trend is found with the magnitudes in either $B, \mathrm{NUV}$, or FUV bands). A steep IMF in LSB galaxies is also suggested by Lee et al. (2004), who found that it provides a better agreement 


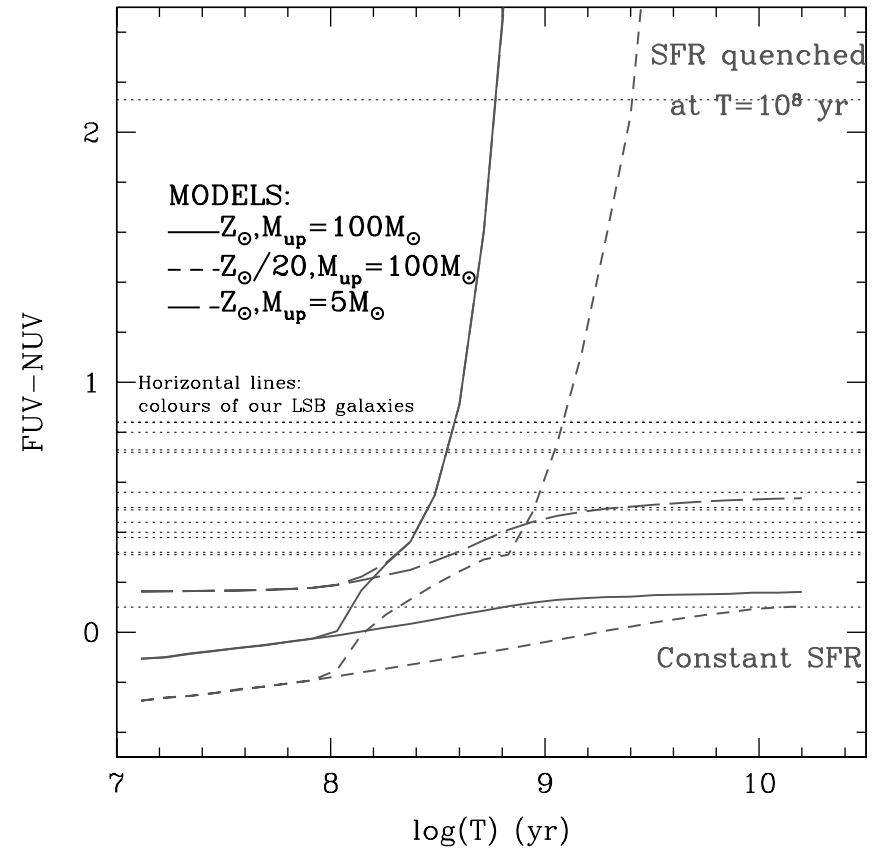

FIG. 6.-Effect of the age or IMF on the FUV-NUV color. Horizontal lines correspond to the values measured in our sample, the various curves to different models as indicated in the figure. [See the electronic edition of the Journal for a color version of this figure.]

with observations of mass-to-light ratios and optical colors $(B-R, B-V, B-I)$. However, their result is based on a single burst scenario (a few Gyr old). If the star formation history is more complex, for instance composed of a few Gyr old burst on top of an older underlying stellar population, a standard (stellar) IMF could be accommodated, since the single burst scenario would require an excess of low-mass stars to compensate for the contribution of the most evolved stellar population. Until more complex star formation histories are considered in similar studies, conclusions concerning the IMF should be considered cautiously. Another constraint on the IMF comes from the fact that LSB galaxies follow the Tully-Fisher relationship (McGaugh 2005), which would be difficult to understand if their IMF were extremely different from the one in HSB galaxies.

In order to test the effect of age and IMF on the FUV-NUV color in a very simple way, we used the code of chemical and spectrophotometric galactic evolution of Boissier \& Prantzos (1999) to compute the evolution of this color in two scenarios, a constant star formation rate and a postburst scenario, in which we use a constant star formation rate for $10^{8} \mathrm{yr}$, after which time the star formation rate is quenched. We assume that the FUV and NUV luminosities are then dominated by the fading of the stars created during this event. For each of these scenarios, we made three assumptions: adopting a solar metallicity and the Kroupa et al. (1993) IMF, a low metallicity (a 20th of solar) with the same IMF, and finally a solar metallicity and the Kroupa et al. (1993) IMF but truncated at a very low mass of $5 M_{\odot}$ (in order to test if the IMF of LSB galaxies is truncated at high masses, as suggested by Weidner \& Kroupa 2005). We note that in the case of the postburst scenario, our results slightly depend on the quenching time used in our computation $\left(10^{8} \mathrm{yr}\right)$. However, the differences obtained by varying this parameter (from $10^{7}$ to $10^{10} \mathrm{yr}$ ) are smaller than the ones obtained between the two metallicities considered (one can guess this small dependence on the quenching time by noting that in Fig. 6 the FUV-NUV color for a constant SFR depends little on the age). Thus our results for the two metallicities provide a realistic range for the age of the event, independent of the duration of the star formation event anterior to the quenching. The results are shown in Figure 6, in which the horizontal dotted lines show the colors measured in our LSB galaxies. Unless the IMF is severely truncated, only one galaxy is consistent with ongoing star formation. Note that the models are dust-free, and that the colors obtained for ongoing star formation $(-0.2$ to 0.2$)$ for different metallicities and ages are consistent with the FUV-NUV corrected for extinction of normal star-forming galaxies, as shown in the bottom panel of Figure 3. To explain the colors of most of the LSB galaxies in the absence of dust, we thus need to either use a truncated IMF, or assume that the star formation was quenched for a significant amount of time, between about 0.7 to $1.4 \mathrm{Gyr}$ (assuming a low metallicity) or 0.1 to $0.3 \mathrm{Gyr}$ (for a solar metallicity), this time being an increasing function of the total mass of the galaxy. Again, even if it is quite possible that the star formation proceed by burst episodes with quiet periods in between, or fluctuates significantly (e.g., Vallenari et al. 2005; Boissier et al. 2003; van den Hoek et al. 2000; Gerritsen \& de Blok 1999; O’Neil et al. 1998), it is somewhat strange that the time elapsed after the last burst is longer (redder color, even if this trend is not very strong) for galaxies with larger $\mathrm{H}$ I masses.

Vallenari et al. (2005) shows the SFR history for UGC 5889, obtained from CMD diagrams. They conclude that SFR proceeded in episodes of very low rates $\left(10^{-2} M_{\odot} \mathrm{yr}^{-1}\right)$, spaced by periods of quiescence. However, from their Figure 9, no period of quiescence longer than $\sim 20$ Myr occurred for a dwarf LSB galaxy. It is even more difficult to think of a reason why in more massive LSB galaxies (such as the ones analyzed here, in which stochasticity should have a smaller effect), those periods could last up to $\sim 300$ Myr. The $N$-body simulations of Gerritsen \& de Blok (1999) result in strongly fluctuating star formation histories, also with timescales of about 20 Myr. Boissier et al. (2003) also had to advocate bursts and quiescent phases to explain several observational facts in LSB galaxies, such as the scatter in the Tully-Fisher relation and gas to luminosity ratio, as well as the existence of some red LSB galaxies (O'Neil et al. 1997). According to their model, quiescent phases should be longer (up to $1 \mathrm{Gyr}$ ) to explain these colors. These works suggest that star formation in LSB galaxies or in low-density regions may proceed by bursts followed by quiescent phases; however, the timescales are not in perfect agreement with the ones we need to explain the FUV-NUV colors.

\section{STAR FORMATION HISTORIES OF LSB GALAXIES}

In $\S 4$, we computed the SFR using standard calibrations. However, the FUV - NUV color suggests either an extinction effect, a drastic truncation of the IMF, or that the UV emitting regions are older and older for more massive galaxies (we actually refer to the $\mathrm{H}$ I mass of the galaxies, for which we have measurements).

If the effect is due to extinction, then the SFRs have been underestimated, and the real SFRs in LSB galaxies are similar to that in normal galaxies. However, with such an assumption, we would have to explain the low level of infrared emission, and the other signs of transparency. It would also be hard to explain the other signs of youth usually observed in LSB galaxies (blue colors, young ages, low metallicity).

For the two other assumptions, we can use the same models as in $\S 5.3$. We computed for each of them the FUV and NUV magnitudes corresponding to star formation rates of $1 M_{\odot} \mathrm{yr}^{-1}$ (continuously, or quenched after $10^{8} \mathrm{yr}$ ). The results are given in Figure 7. 

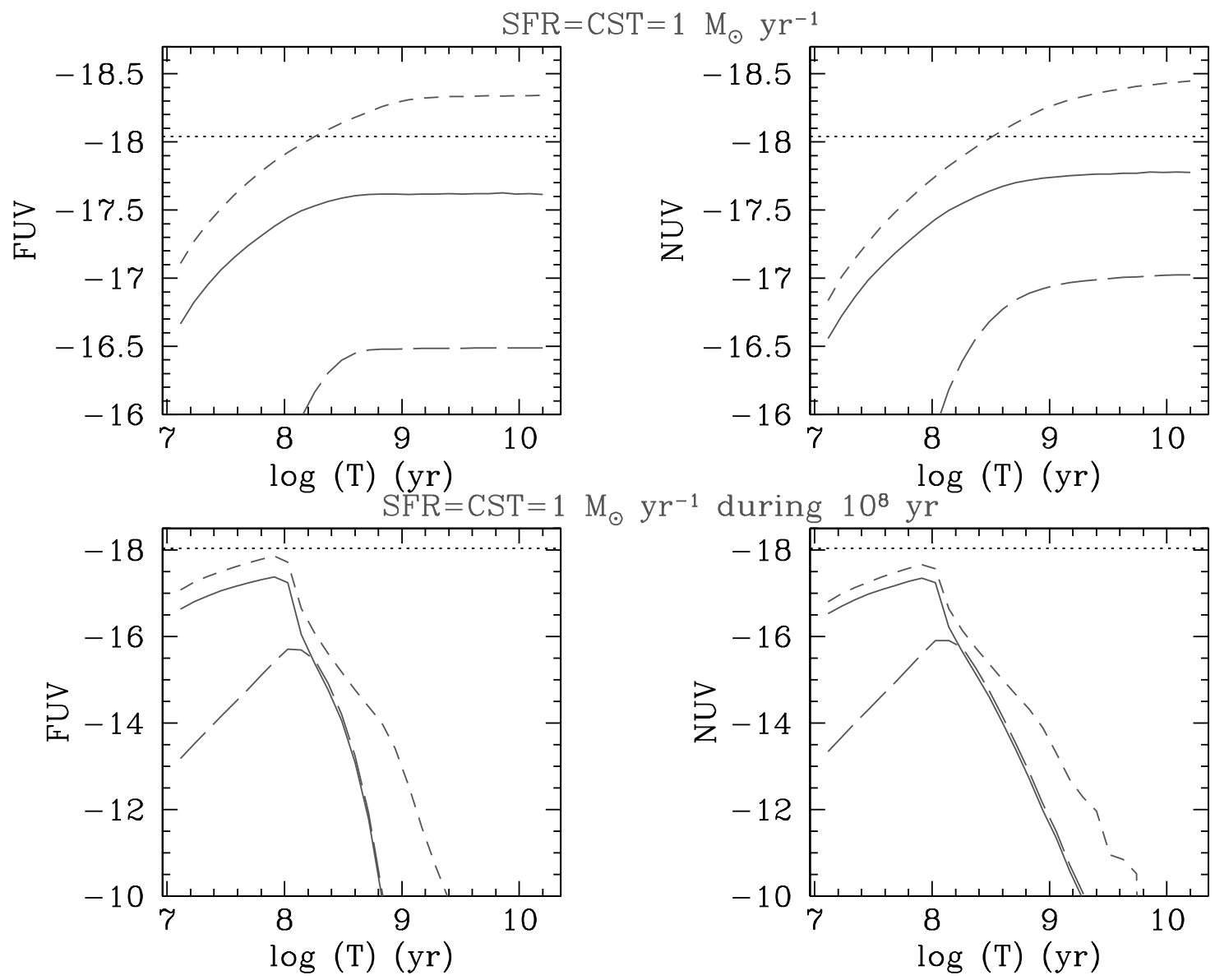

FIG. 7.-FUV and NUV magnitudes for a star formation of $1 M_{\odot} \mathrm{yr}^{-1}$, continuously (top) or quenched after $10^{8} \mathrm{yr}$ (bottom). The horizontal line shows the magnitude obtained using the calibration of Kennicutt (1998). The various curves correspond to the various assumptions concerning the IMF and the metallicity, as presented in Fig. 6. [See the electronic edition of the Journal for a color version of this figure.]

First, for a continuous star formation rate, we find that our models are consistent with the conversion factor given by Kennicutt (1998). However, depending on the metallicity, deviations with respect to this calibration up to about $\sim 0.5$ mag are observed (a factor of $\sim 1.5$ on the SFR).

If we adopt the severe truncation at $5 M_{\odot}$ on the IMF, we predict of course a much weaker UV emission, by 1.5 mag in FUV and about $1 \mathrm{mag}$ in NUV. If this is true, then our NUV SFRs were underestimated by a factor of about 3 . In that case, the star formation rate at a given $\mathrm{H}$ I mass (or star formation efficiency) would be barely lower in LSB galaxies than in "normal" galaxies.

As mentioned above, several works have suggested that the star formation in LSB galaxies proceeds in a sequence of bursts and quiescent phases (Vallenari et al. 2005; Gerritsen \& de Blok 1999; Boissier et al. 2003). We try in the following to see what we can conclude concerning the star formation history of LSB and low-density regions under this assumption. We should also mention the study of Boquien et al. (2007), who discussed various star formation indicators (including the UV) in low-density $\mathrm{H}$ i probably expelled during a galactic collision. They found that the UV actually overestimates the current value of the SFR, but in that case the current value of the SFR is not very pertinent, as the SFR was much higher shortly after the collision.

Adopting the curves at the bottom of Figure 7, and the one in Figure 6 for the case of quenched SFR, we can compute from the FUV-NUV color the time elapsed after the quenching, and then from the NUV evolution given in Figure 7 the level of SFR during the active phase. We do this for both the solar and low- metallicity $\left(Z_{\odot} / 20\right)$ cases in order to get an idea of the uncertainty due to the metallicity.

For each galaxy, we also compute the more plausible value by fitting the luminosity-metallicity relationship in McGaugh $\left(1994 ; \log Z / Z_{\odot}=-2.81-0.11 M_{B}\right)$, and use the metallicity obtained in that way $\left(Z_{M_{B}}\right)$. Due to the paucity of measurements of abundances in LSB galaxies, this value is, however, quite uncertain. The results are given in Table 4 . We also show the values derived as a function of the $\mathrm{H}$ I mass (see Fig. 8). Our galaxies are characterized by time elapsed since the last burst, of a few hundred million years up to more than $1 \mathrm{Gyr}$, and very high star formation rates (at least for galaxies more massive than $10{ }^{10} M_{\odot}$ in $\mathrm{H}_{\mathrm{I}}$ ) during the active phase. The actual number of stars formed depends on how long this active phase lasted.

The amplitude of the bursts suggested by our results are quite large with respect to the one found in, e.g., Gerritsen \& de Blok (1999) or Vallenari et al. (2005): a few $M_{\odot} \mathrm{yr}^{-1}$ for the dwarf LSB galaxies, up to several hundred $M_{\odot} \mathrm{yr}^{-1}$ in massive LSB galaxies. On the other hand, O'Neil et al. (1998) show that the present SFR in LSB galaxies is too small to produce their total luminosity, suggesting that the SFR has been much larger in the past. We note that some of the parameters involved in the computation of the theoretical FUV-NUV color are uncertain. This could have some effect on the elapsed time and burst amplitudes derived. Also, the burst could be extended in time (instead of an instantaneous quenching), making the evolution of the FUV luminosity shown in Figure 7 less abrupt. This could make our elapsed time and amplitude bursts compatible with the results of the works of Gerritsen \& de Blok (1999), Vallenari et al. (2005), 
TABLE 4

Time Elapsed Since Last Burst and Star Formation Rates DURING THE BURST

\begin{tabular}{|c|c|c|c|c|c|c|}
\hline \multirow[b]{2}{*}{ Name } & \multicolumn{3}{|c|}{$\begin{array}{c}\text { Time Elapsed } \\
\text { (Gyr) }\end{array}$} & \multicolumn{3}{|c|}{$\begin{array}{c}\mathrm{SFR}_{\text {burst }} \\
\left(M_{\odot} \mathrm{yr}^{-1}\right)\end{array}$} \\
\hline & Low $Z$ & $Z_{M_{B}}$ & High $Z$ & Low $Z$ & $Z_{M_{B}}$ & High $Z$ \\
\hline UGC $00568 .$. & 2.453 & 1.353 & 0.487 & 33.327 & 24.947 & 11.415 \\
\hline UGC $01230 \ldots \ldots \ldots . .$. & 0.583 & 0.413 & 0.108 & 13.873 & 11.166 & 5.196 \\
\hline UGC $02936 \ldots \ldots \ldots . .$. & & & & 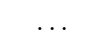 & $\ldots$ & \\
\hline OBC $97-\mathrm{C} 04-2 \ldots \ldots$ & 1.114 & 1.057 & 0.278 & 3.550 & 3.362 & 1.479 \\
\hline F564-V3 …........... & 0.106 & 0.108 & 0.025 & 0.010 & 0.010 & 0.005 \\
\hline UGC $05209 \ldots \ldots \ldots$ & 0.724 & 0.732 & 0.163 & 0.251 & 0.245 & 0.109 \\
\hline F568-1 …................... & 0.674 & 0.607 & 0.148 & 16.437 & 15.530 & 7.032 \\
\hline F568-3 ...................... & 0.565 & 0.449 & 0.102 & 13.921 & 11.947 & 5.125 \\
\hline UGC $05750 \ldots \ldots \ldots . .$. & 0.650 & 0.583 & 0.141 & 12.367 & 11.587 & 5.262 \\
\hline PGC $135754 \ldots \ldots \ldots$ & 1.080 & 0.782 & 0.267 & 139.026 & 105.025 & 57.804 \\
\hline F568-6 _................... & $\ldots$ & $\ldots$ & $\ldots$ & $\ldots$ & $\ldots$ & $\ldots$ \\
\hline F571-V1 ................... & 0.794 & 0.752 & 0.186 & 8.056 & 7.818 & 3.570 \\
\hline Malin 1...................... & 1.114 & 0.597 & 0.278 & 225.283 & 142.340 & 93.836 \\
\hline PGC $45080 \ldots \ldots \ldots . . . .$. & $\ldots$ & & & & & \\
\hline F530-1 …................. & 1.016 & 0.799 & 0.249 & 174.621 & 143.097 & 73.402 \\
\hline F533-3 ..................... & 0.847 & 0.630 & 0.209 & 93.536 & 77.953 & 42.575 \\
\hline NGC $7589 \ldots \ldots \ldots . . . .$. & 0.785 & 0.466 & 0.182 & 52.945 & 39.502 & 23.342 \\
\hline PGC $71626 \ldots \ldots \ldots$. & 1.005 & 0.716 & 0.247 & 241.341 & 187.828 & 102.281 \\
\hline
\end{tabular}

NoтE $-Z$ indicates the metallicity adopted. High and low metallicity are respectively solar and $1 / 20$ solar metallicities. $Z_{M_{B}}$ is the metallicity deduced from the metallicity-luminosity relationship in McGaugh (1994).

or van den Hoek et al. (2000), which address relatively low-mass LSB galaxies. It would be hard, however, to sufficiently diminish the $\mathrm{SFR}_{\text {burst, }}$ derived in giant LSB galaxies to change the trends seen in Figure 7: galaxies more massive than about $10^{10} M_{\odot}$ (in H I) show larger SFRs and elapsed time since the last burst than less massive ones (even if the latter trend is unclear when adopting the more plausible metallicity). In this context, we should note that Mapelli et al. (2008) suggested that ring galaxies (such as the Cartwheel) may evolve into giant LSB galaxies like Malin 1 in their late stage (about 500 Myr after a collision), what

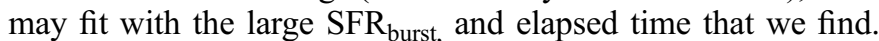
However, their analysis did not include UV data or predictions for this wavelength.

With the elapsed time that we find, one could expect to find almost no $\mathrm{H} \alpha$ emission in LSB galaxies, while it is commonly observed (e.g., references in Table 3 or the rotation curves of McGaugh et al. 2001; Swaters et al. 2000). However, many of these $\mathrm{H} \alpha$ detections concern relatively low luminosities (Swaters et al. 2000) or dwarf (van Zee et al. 1997) LSB galaxies, while the elapsed times we find are significantly large only in the more massive LSB galaxies, in which the presence of $\mathrm{H} \alpha$ would pose stronger constraints. Also, our method based on the FUV-NUV color is able to date the last significant event contributing to the UV spectra. Smaller and more recent star formation could have occurred, as long as the UV emission is still dominated by the older event (This would also lead to deriving lower SFR from $\mathrm{H} \alpha$ than from the UV). Finally, the quenching could not be instantaneous, but rather presents a smooth decline (we do not attempt to model it to avoid the multiplication of free parameters), making $\mathrm{H} \alpha$ visible for a longer time.

In the following, we will estimate the total duration of the active phases needed to form all the stars in these galaxies, given SFR $_{\text {burst }}$. Assuming an average $K$-band mass-to-light ratio of $\log (M / L)=-0.3$ (Bell \& de Jong 2001), we can compute the stellar mass from the $K$ band total magnitude for galaxies with

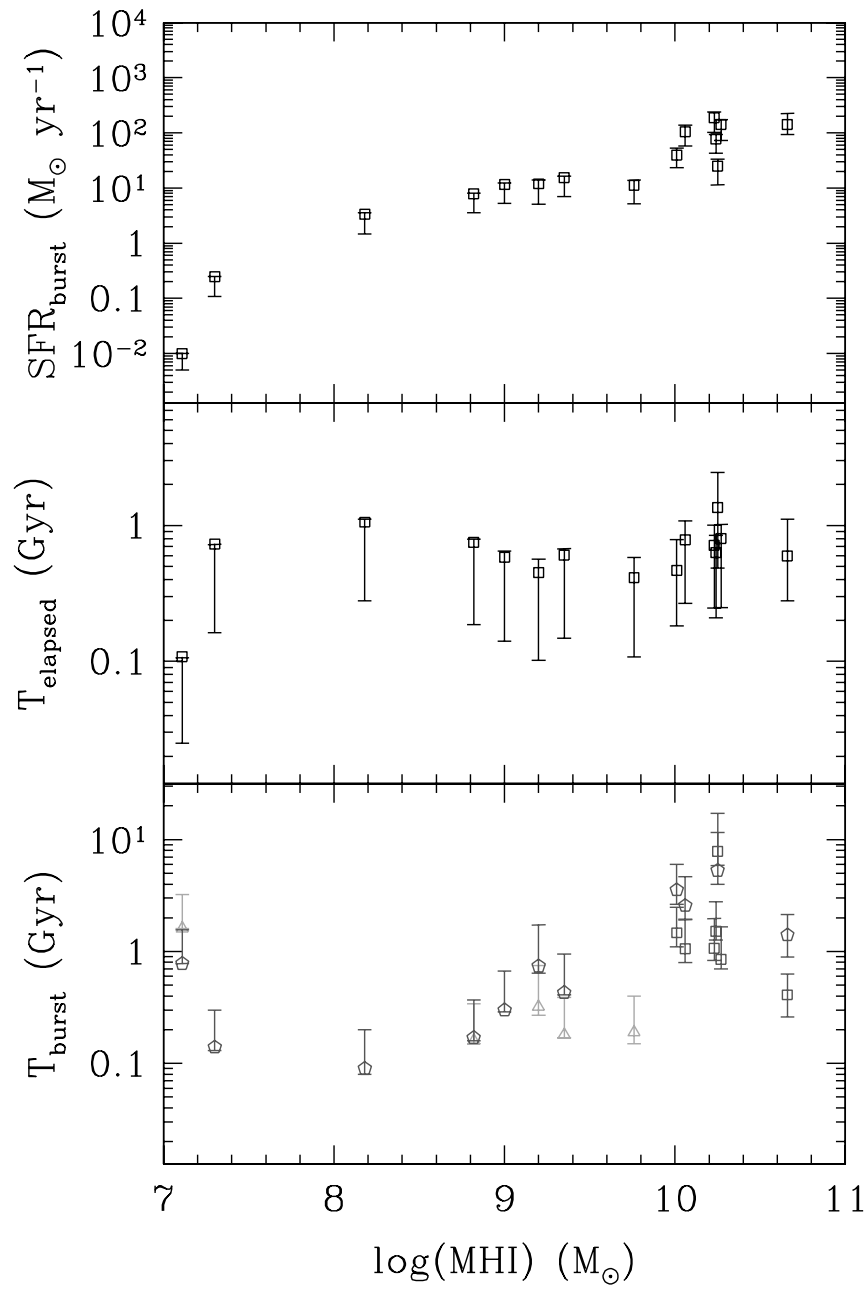

FIG. 8.-SFR during the star formation event (top), time elapsed since the last star formation event (middle), and time necessary to build up the total stellar mass of the galaxy (bottom) as a function of the $\mathrm{H}$ I mass. The points show values derived assuming the metallicity-luminosity relationship of McGaugh (1994). The error bars indicates values obtained when the metallicity is moved within the range $Z_{\odot} / 20$ to $Z_{\odot}$, encompassing values found in LSB galaxies. In the bottom panel, squares, triangles, and pentagons correspond to adopting the stellar masses derived from $K, R$, and $r$ band magnitudes, respectively. [See the electronic edition of the Journal for a color version of this figure.]

2MASS data. For galaxies in de Blok et al. (1995) we can compute a stellar mass from the $R$-band magnitude, with the stellar mass-to-light ratio of Bell \& de Jong (2001) depending on $B-R$. From the SDSS $r$-band data, we can also compute a stellar mass adopting the average trend shown in Kauffmann et al. (2003). These stellar masses are given in Table 5. They are rough estimates, with the differences between the various masses giving an idea of the uncertainty $(\sim 0.2$ dex $)$.

Assuming that the star formation proceed by bursts of amplitude $\mathrm{SFR}_{\text {burst }}$ (Table 4), we determined how long it takes to produce the whole stellar mass of the galaxy $\left[T_{\text {burst }}=M_{*} /(1-\right.$ $\left.R) \mathrm{SFR}_{\text {burst }}\right]$, where $R$ is the returned fraction, for which we adopt $R=0.3$, corresponding to the Kroupa et al. (1993) IMF. The values obtained (for each estimation of the stellar mass, and for the SFR determined for various metallicities) are given in Table 5 and Figure 8. The "burst" time is actually the total time during which the galaxy had to be in an active phase to build up its stellar mass (assuming no stars at all were formed during the quiescent periods), even if it may have been split in several events of similar SFR burst. 
TABLE 5

Stellar Mass and Active Phase Duration

\begin{tabular}{|c|c|c|c|c|c|c|c|c|c|c|c|c|}
\hline \multirow[b]{2}{*}{ NAME } & \multicolumn{4}{|c|}{$K$ BAND } & \multicolumn{4}{|c|}{$R$ BAND } & \multicolumn{4}{|c|}{$r$ BAND } \\
\hline & $\begin{array}{c}\log M_{*}(K) \\
\left(M_{\odot}\right)\end{array}$ & $Z_{\odot} / 20$ & $Z_{M_{B}}$ & $Z_{\odot}$ & $\begin{array}{c}\log M_{*}(R) \\
\left(M_{\odot}\right)\end{array}$ & $Z_{\odot} / 20$ & $Z_{M_{B}}$ & $Z_{\odot}$ & $\begin{array}{c}\log M_{*}(r) \\
\left(M_{\odot}\right)\end{array}$ & $Z_{\odot} / 20$ & $Z_{M_{B}}$ & $Z_{\odot}$ \\
\hline 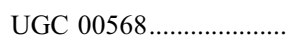 & 11.14 & 5.88 & 7.86 & 17.18 & $\ldots$ & $\ldots$ & $\ldots$ & $\ldots$ & 10.97 & 3.97 & 5.30 & 11.58 \\
\hline 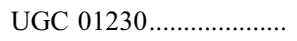 & $\ldots$ & $\ldots$ & $\ldots$ & $\ldots$ & 9.16 & 0.15 & 0.19 & 0.40 & $\ldots$ & $\ldots$ & $\ldots$ & $\ldots$ \\
\hline 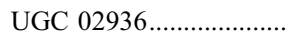 & $\ldots$ & $\ldots$ & $\ldots$ & $\ldots$ & $\ldots$ & $\ldots$ & $\ldots$ & $\ldots$ & $\ldots$ & $\ldots$ & $\ldots$ & $\ldots$ \\
\hline 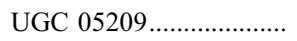 & $\ldots$ & $\ldots$ & $\ldots$ & $\ldots$ & $\ldots$ & $\ldots$ & $\ldots$ & $\ldots$ & 7.37 & 0.13 & 0.14 & 0.30 \\
\hline 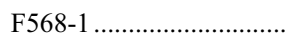 & $\ldots$ & $\ldots$ & $\ldots$ & $\ldots$ & 9.28 & 0.17 & 0.18 & 0.39 & 9.67 & 0.41 & 0.43 & 0.95 \\
\hline F568-3 ........................... & $\ldots$ & $\ldots$ & $\ldots$ & $\ldots$ & 9.43 & 0.27 & 0.32 & 0.75 & 9.79 & 0.64 & 0.74 & 1.73 \\
\hline UGC $05750 \ldots \ldots \ldots \ldots \ldots \ldots$ & $\ldots$ & $\ldots$ & $\ldots$ & $\ldots$ & $\ldots$ & $\ldots$ & $\ldots$ & $\ldots$ & 9.39 & 0.29 & 0.30 & 0.67 \\
\hline 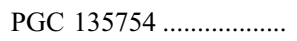 & 10.89 & 0.80 & 1.06 & 1.92 & $\ldots$ & $\ldots$ & $\ldots$ & $\ldots$ & 11.28 & 1.94 & 2.57 & 4.66 \\
\hline 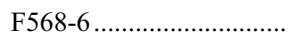 & $\ldots$ & $\ldots$ & $\ldots$ & $\ldots$ & $\ldots$ & $\ldots$ & $\ldots$ & $\ldots$ & $\ldots$ & $\ldots$ & $\ldots$ & 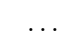 \\
\hline F533-3 _................................. & 10.92 & 1.27 & 1.52 & 2.78 & $\ldots$ & $\ldots$ & $\ldots$ & $\ldots$ & $\ldots$ & $\ldots$ & $\ldots$ & $\ldots$ \\
\hline 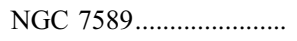 & 10.61 & 1.10 & 1.47 & 2.49 & $\ldots$ & $\ldots$ & $\ldots$ & $\ldots$ & 10.99 & 2.65 & 3.55 & 6.01 \\
\hline 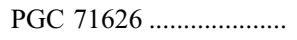 & 11.15 & 0.83 & 1.07 & 1.97 & $\ldots$ & $\ldots$ & $\ldots$ & $\ldots$ & $\ldots$ & $\ldots$ & $\ldots$ & $\ldots$ \\
\hline
\end{tabular}

Nоте.-Stellar mass derived from various photometric bands (see text), and burst duration obtained from it, adopting various metallicities.

From Figure 8, we can see that the results are quite dispersed, due to the approximations we have to make to derive SFR and stellar masses. However, it seems that above $10^{10} M_{\odot}$ (corresponding to galaxies in which we find very high $\mathrm{SFR}_{\text {burst }}$ ), the time elapsed after the last burst increases with the mass. At the same time, $T_{\text {burst }}$, the time during which the galaxy was in a "burst" phase during its history, decreases with the mass (this result is, however, very uncertain, especially due to the uncertainty on the metallicity, affecting $T_{\text {elapsed }}$ and the quantities derived from it). This opposite behavior of $T_{\text {elapsed }}$ and $T_{\text {burst }}$ is indeed expected when the fraction of "active phase" duration with respect to the life of galaxy gets smaller; there is less and less chance to catch the galaxy during this phase (for galaxies with $T_{\text {burst }} \sim 1 \mathrm{Gyr}$, chances are already lower than $10 \%$ ), and more and more chances to find a galaxy with larger elapsed time.

\section{CONCLUSIONS}

In this paper, we presented the GALEX UV observations of 18 low surface brightness galaxies, with known $\mathrm{H}$ i content. The UV light relative to the optical is more extended than in normal star-forming galaxies. Morphologies similar to extended UV disks (XUV) are often found (about 70\% of our objects; F568-3 [Malin 2] is a very nice example), although not systematically.

Adopting standard calibrations to convert the UV light into star formation rates, we obtain a large range of SFR (a few $10^{-3}$ to a few solar masses per year), depending on the $\mathrm{H}$ I mass of the galaxy. Massive LSB galaxies have lower SFR than normal star-forming galaxies with the same gas reservoir (by a factor of $\sim 5$ ). Such a lower efficiency for forming stars is expected in various models, where, e.g., LSB galaxies a have larger radial extent due to larger spin parameters. However, the SFRs obtained in such a way are highly uncertain, due to the very red FUV-NUV color (especially in massive LSB galaxies) that we measure, which might indicate a highly non-constant star formation history.
Several interpretations for this color are possible, and more work on stellar populations in LSB galaxies is needed to obtain a definitive answer. The various possibilities we considered are:

1. A dust attenuation effect. Note, however, that the low infrared emission found in LSB galaxies makes this explanation unlikely.

2. Variations in the initial mass function. The FUV-NUV colors can be recovered in a constant star formation scenario, but only for an extremely steep (or truncated) IMF. Moreover, we find that the more massive galaxies (as measured with the $\mathrm{H}$ I mass) are redder, while the IMF proposed by Weidner \& Kroupa (2005), depending on the star formation rate, should have the opposite effect. We do not exclude the possibility that an IMF effect might play a role in galaxies with low $\mathrm{H}$ I masses (and thus low star formation rates), but it cannot be responsible for the trends seen in the more massive galaxies.

3. An age effect. Red FUV-NUV colors can be obtained if the SFR was quenched. In this scenario, we find that the more massive LSB galaxies have known more massive star formation events (larger $\mathrm{SFR}_{\text {burst }}$, followed by relatively long quiescent phases of several hundred million years). If these events are spread over the whole history of the galaxy, on average the SFR can be about constant over the Gyr timescale, giving as a result optical-near-infrared colors similar to late-type galaxies (as those observed), while the FUV-NUV can get relatively red during the quiescent phase, dominating the lifetime of the galaxy. To explain the fact that most massive galaxies are redder, it would be necessary to assume that stars in these galaxies are formed during bursts of larger amplitudes, separated by longer quiescent epochs. The more extreme example of this effect is UGC 00568, for which the quiescent phase could have lasted up to 2 Gyr (also affecting the optical colors in this case; indeed, its SED is typical of early-type galaxies), while a huge amount of $\mathrm{H}_{\mathrm{I}}$ is present. A clear physical reason for this is still to be found. We can only speculate that massive LSB galaxies have large gas reservoirs, in which star formation is suddenly turned on only 
occasionally. The next large burst event cannot occur until the gas reservoir has been built up again.

In any case, it seems quite dangerous to derive SFRs from the UV light in low-density regions (LSB galaxies, outer XUV disks) without taking some precautions, especially if red FUV-NUV colors are observed or if there is no independent way to date the star formation event.

We thank the referee, Stacy McGaugh, for his very constructive comments, as well as David Thilker and Ted Wyder for dis- cussions. This research is first based on the GALEX GI program GALEXGII04-0000-0067, as well as public archival data, available at the MAST archive. This research has made use of the NASA/IPAC Extragalactic Database (NED) which is operated by the Jet Propulsion Laboratory, California Institute of Technology, under contract with the National Aeronautics and Space Administration. We acknowledge the usage of the HyperLeda database (http://leda.univ-lyon1.fr). This research has made use of the GOLD Mine Database.

Facilities: GALEX

\section{APPENDIX}

\section{GALEX FUV AND NUV IMAGES}

Figure Set 9 shows the GALEX FUV and NUV images, as well as the SDSS $g$ image when available (replaced by the DSS-1 red image when it is not). The bottom right panel shows the broadband SED (in AB magnitudes) constructed from UV-optical-nearinfrared photometry when available, including GALEXUV, optical data from the SDSS and the literature, and 2MASS near-infrared (see text for details).
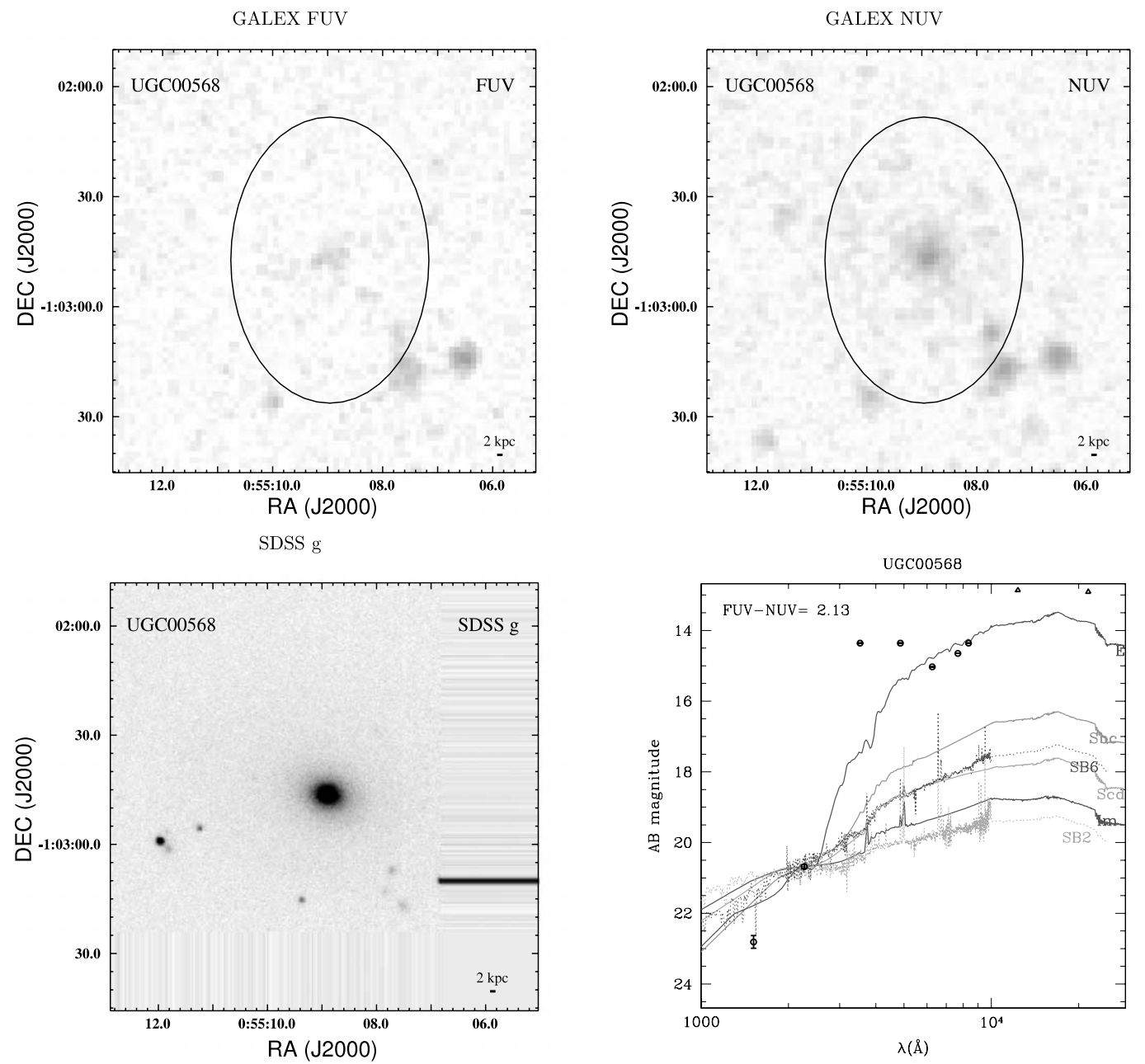

FIG. 9.1. UGC 00568

FIG. SET 9.-- Images and SED (bottom right). GALEX and SDSS magnitudes were computed in this paper; other wavelengths are taken from NED and the literature (see text). The name of the galaxy is indicated in each panel. Ellipses indicate the optical diameter (2a), for the adopted inclination and P.A. (see parameters in Table 1). In the bottom right panel, circles with error bars show our photometry of GALEX and SDSS data. Triangles correspond to data from 2MASS, squares to values from Hunter \& Elmegreen (2006), crosses to values from de Blok et al. (1995), and diamonds to values from McGaugh \& Bothun (1994). Templates in the bottom right panel are taken from Coleman et al. (1980, with the extrapolation of Arnouts et al. 1999) and Kinney et al. (1993) as distributed in Le Phare (http://www.lam.oamp.fr/arnouts/ LE_PHARE.html). [See the electronic edition of the Journal for Figs. 9.2-9.18.] 
REFERENCES

Arnouts, S., Cristiani, S., Moscardini, L., Matarrese, S., Lucchin, F., Fontana, A., \& Giallongo, E. 1999, MNRAS, 310, 540

Barth, A. J. 2007, AJ, 133, 1085

Bell, E. F., Barnaby, D., Bower, R. G., de Jong, R. S., Harper, D. A., Hereld, M., Loewenstein, R. F., \& Rauscher, B. J. 2000, MNRAS, 312, 470

Bell, E. F., \& de Jong, R. S. 2001, ApJ, 550, 212

Bianchi, L., et al. 2005, ApJ, 619, L71

Boissier, S., Monnier Ragaigne, D., Prantzos, N., van Driel, W., Balkowski, C., \& O’Neil, K. 2003, MNRAS, 343, 653

Boissier, S., \& Prantzos, N. 1999, MNRAS, 307, 857 2000, MNRAS, 312, 398

Boissier, S., et al. 2007, ApJS, 173, 524

Boquien, M., Duc, P.-A., Braine, J., Brinks, E., Lisenfeld, U., \& Charmandaris, V. 2007, A\&A, 467, 93

Boselli, A., Boissier, S., Cortese, L., Gil de Paz, A., Seibert, M., Madore, B. F., Buat, V., \& Martin, D. C. 2006, ApJ, 651, 811

Boselli, A., Gavazzi, G., \& Sanvito, G. 2003, A\&A, 402, 37

Boselli, A., Lequeux, J., \& Gavazzi, G. 2002, A\&A, 384, 33

Boselli, A., et al. 2005, ApJ, 623, L13

Bothun, G., Impey, C., \& McGaugh, S. 1997, PASP, 109, 745

Buat, V., \& Xu, C. 1996, A\&A, 306, 61

Buat, V., et al. 2005, ApJ, 619, L51

Burkholder, V., Impey, C., \& Sprayberry, D. 2001, AJ, 122, 2318

Chung, A., van Gorkom, J. H., O’Neil, K., \& Bothun, G. D. 2002, AJ, 123, 2387

Coleman, G. D., Wu, C.-C., \& Weedman, D. W. 1980, ApJS, 43, 393

Cortese, L., et al. 2006, ApJ, 637, 242 2008, MNRAS, submitted

Dalcanton, J. J., Spergel, D. N., \& Summers, F. J. 1997, ApJ, 482, 659

Dale, D. A., \& Helou, G. 2002, ApJ, 576, 159

Dale, D. A., Helou, G., Contursi, A., Silbermann, N. A., \& Kolhatkar, S. 2001, ApJ, 549, 215

Das, M., O’Neil, K., Vogel, S. N., \& McGaugh, S. 2006, ApJ, 651, 853

de Blok, W. J. G., McGaugh, S. S., \& van der Hulst, J. M. 1996, MNRAS, 283, 18 de Blok, W. J. G., van der Hulst, J. M., \& Bothun, G. D. 1995, MNRAS, 274, 235

Gavazzi, G., Boselli, A., Donati, A., Franzetti, P., \& Scodeggio, M. 2003, A\&A, 400, 451

Gavazzi, G., Boselli, A., van Driel, W., \& O’Neil, K. 2005, A\&A, 429, 439

Gerritsen, J. P. E., \& de Blok, W. J. G. 1999, A\&A, 342, 655

Gil de Paz, A., et al. 2005, ApJ, 627, L29

$$
\text { 2007a, ApJ, 661, } 115
$$$$
\text { 2007b, ApJS, 173, } 185
$$

Gordon, K. D., Clayton, G. C., Witt, A. N., \& Misselt, K. A. 2000, ApJ, 533, 236

Heckman, T., et al. 1995, ApJ, 452, 549

Hinz, J. L., Rieke, M. J., Rieke, G. H., Willmer, C. N. A., Misselt, K., Engelbracht, C. W., Blaylock, M., \& Pickering, T. E. 2007, ApJ, 663, 895

Holwerda, B. W., Gonzalez, R. A., Allen, R. J., \& van der Kruit, P. C. 2005, AJ, 129,1396

Hunter, D. A., \& Elmegreen, B. G. 2006, ApJS, 162, 49
Jansen, R. A., Fabricant, D., Franx, M., \& Caldwell, N. 2000, ApJS, 126, 331 Jimenez, R., Padoan, P., Matteucci, F., \& Heavens, A. F. 1998, MNRAS, 299, 123

Kauffmann, G., et al. 2003, MNRAS, 341, 33

Kennicutt, R. C. 1998, ARA\&A, 36, 189

Kinney, A. L., Bohlin, R. C., Calzetti, D., Panagia, N., \& Wyse, R. F. G. 1993, ApJS, 86, 5

Kong, X., Charlot, S., Brinchmann, J., \& Fall, S. M. 2004, MNRAS, 349, 769 Kroupa, P., Tout, C. A., \& Gilmore, G. 1993, MNRAS, 262, 545

Lee, H.-c., Gibson, B. K., Flynn, C., Kawata, D., \& Beasley, M. A. 2004, MNRAS, 353, 113

Mapelli, M., Moore, B., Ripamonti, E., Mayer, L., Colpi, M., \& Giordano, L. 2008, MNRAS, 383, 1223

Martin, C. L., \& Kennicutt, R. C., Jr. 2001, ApJ, 555, 301

Matthews, L. D., van Driel, W., \& Monnier-Ragaigne, D. 2001, A\&A, 365, 1

McGaugh, S. S. 1992, Ph.D. thesis, Univ. Michigan 1994, ApJ, 426, 135 2005, ApJ, 632, 859

McGaugh, S. S., \& Bothun, G. D. 1994, AJ, 107, 530

McGaugh, S. S., Rubin, V. C., \& de Blok, W. J. G. 2001, AJ, 122, 2381

Meurer, G. R., Heckman, T. M., \& Calzetti, D. 1999, ApJ, 521, 64

Meurer, G. R., Heckman, T. M., Leitherer, C., Kinney, A., Robert, C., \& Garnett, D. R. 1995, AJ, 110, 2665

O’Neil, K., Bothun, G. D., \& Schombert, J. 1998, AJ, 116, 2776

O’Neil, K., Bothun, G. D., Schombert, J., Cornell, M. E., \& Impey, C. D. 1997, AJ, 114, 2448

O’Neil, K., Oey, M. S., \& Bothun, G. 2007, AJ, 134, 547

O’Neil, K., \& Schinnerer, E. 2004, ApJ, 615, L109

Panuzzo, P., Bressan, A., Granato, G. L., Silva, L., \& Danese, L. 2003, A\&A, 409, 99

Paturel, G., Petit, C., Prugniel, P., Theureau, G., Rousseau, J., Brouty, M., Dubois, P., \& Cambrésy, L. 2003, A\&A, 412, 45

Rahman, N., Howell, J. H., Helou, G., Mazzarella, J. M., \& Buckalew, B. 2007, ApJ, 663, 908

Sancisi, R., \& Fraternali, F. 2007, in Proc. 41st ESLAB Symp., The Impact of HST on European Astronomy (Noordwijk: ESA), in press (arXiv:0707.2377)

Seibert, M., et al. 2005, ApJ, 619, L55

Swaters, R. A., Madore, B. F., \& Trewhella, M. 2000, ApJ, 531, L107

Thilker, D., et al. 2007, ApJS, 173, 572

Thilker, D. A., et al. 2005, ApJ, 619, L79

Vallenari, A., Schmidtobreick, L., \& Bomans, D. J. 2005, A\&A, 435, 821

van den Hoek, L. B., de Blok, W. J. G., van der Hulst, J. M., \& de Jong, T. 2000, A\&A, 357, 397

van der Hulst, J. M., Skillman, E. D., Smith, T. R., Bothun, G. D., McGaugh, S. S., \& de Blok, W. J. G. 1993, AJ, 106, 548

van Zee, L., Haynes, M. P., Salzer, J. J., \& Broeils, A. H. 1997, AJ, 113, 1618

Weidner, C., \& Kroupa, P. 2005, ApJ, 625, 754

Zaritsky, D., \& Christlein, D. 2007, AJ, 134, 135 